\title{
Remarkably Mild and Simple Preparation of Sulfenate Anions from $\beta$-Sulfinylesters: A New Route to Enantioenriched Sulfoxides
}

Caroline Caupène, Cédric Boudou, Stéphane Perrio,* and Patrick Metzner

Laboratoire de Chimie Moléculaire et Thio-organique (UMR CNRS 6507), ENSICaen-Université de Caen Basse-Normandie, 6 Boulevard du Maréchal Juin, F-14050 Caen (France)

e-mail: stephane.perrio@ensicaen.fr

\section{Supporting Information}

General Conditions

Typical Procedure and Spectral Data of $\beta$-Sulfanylesters 4

Typical Procedure and Spectral Data of $\beta$-Sulfinylesters 1

Esters 3

HPLC Analyses 


\section{General Conditions}

Dry THF, diethyl ether and toluene were displayed by a passage down an activated alumina column. Cumene, pentane and 2,2,5,5-tetramethyltetrahydrofuran were freshly distilled under a nitrogen atmosphere over $\mathrm{CaH}_{2}$. All other reagents and solvents were used as received from commercial sources. All reactions were performed in oven-dried glassware, under an atmosphere of dry nitrogen. Low reaction temperatures stated were those of the reaction mixture and were maintained constant using a cooler. The concentrations of organolithium reagents were determined by titration against diphenylacetic acid. ${ }^{1}$ Reactions were purified by chromatography column with silica gel Si $60(0.040-0.063 \mathrm{~nm})$. Thin layer chromatography was carried out on silica gel $60 \mathrm{~F}_{254}(1.1 \mathrm{~mm})$ with spot detection under UV light or through $\mathrm{I}_{2}$ or $\mathrm{KMnO}_{4}$ oxidation. Melting points were obtained on a capillary apparatus and are uncorrected. All chemical shifts ( $\delta$ ) and coupling constants are quoted in parts per million (ppm) and Hertz (Hz) respectively. The following abbreviations are used to designate the multiplicity of the signals: $\mathrm{s}=$ singlet; $\mathrm{d}=$ doublet; $\mathrm{t}=$ triplet $; \mathrm{q}=$ quartet $; \mathrm{m}=$ multiplet, and combinations thereof. The chemical shifts are calibrated to TMS $(\delta \mathrm{H} 0.00)$ or residual proton and carbon resonance of the solvents $\mathrm{CDCl}_{3}(\delta \mathrm{H}$ 7.26 and $\delta \mathrm{C} 77.16)$. IR spectra were recorded on a FT-IR instrument. Mass spectra were recorded on a GC/MS/MS instrument. Only peaks of an intensity $>10 \%$ (except decisive ones) are listed.

Enantiomeric excesses were determined by chiral HPLC equipped with a photodiode array detector $(200-300 \mathrm{~nm})$ and one of the following columns: $A D$ or $A D-H(0.46 \mathrm{~cm} \times 25 \mathrm{~cm})$ and $O B$ or $O B-H(0.46 \mathrm{~cm} \times 25 \mathrm{~cm})$. In all cases, the analysis was calibrated with a sample of the racemate. Racemic references of 1-benzylsulfinyl-4-methylbenzene $\mathbf{2} \mathbf{a}_{\mathbf{1}}$ and 1-methylsulfinyl-4-

(1) Kofron, W. G.; Baclawski, L. M. J. Org. Chem. 1976, 41, 1879-1880. 
methylbenzene $\mathbf{2} \mathbf{a}_{2}$ were prepared by $S$-alkylation of the corresponding lithium sulfenate salts prepared by the thiolate oxidation methodology previously described by us. ${ }^{2}$ Enantioenrichied references of the same sulfoxides with a major $(S)$-configuration were prepared by oxidation of the corresponding thioether with commercial $(+)$-(8,8-dichlorocamphorylsulfonyl)oxaziridine under reported conditions. ${ }^{3}$ Accordingly, $(\boldsymbol{S})-\mathbf{2} \mathbf{a}_{2}\left(\mathrm{R}^{1}=4-\mathrm{MeC}_{6} \mathrm{H}_{5}\right.$ and $\left.\mathrm{R}^{3}=\mathrm{Me}\right)$ was obtained in $90 \%$ yield and $66 \%$ ee whilst $(S)-2 \mathrm{a}_{1}\left(\mathrm{R}^{1}=4-\mathrm{MeC}_{6} \mathrm{H}_{5}\right.$ and $\left.\mathrm{R}^{3}=\mathrm{Bn}\right)$ was formed in $75 \%$ ee. The isolated yield in sulfoxide $(\boldsymbol{S}) \mathbf{- 2} \mathbf{a}_{\mathbf{1}}$ is not given because of contamination with $\mathrm{N}$-sulfonylimine derived from the oxidizing reagent.

$\beta$-Sulfanylester $\mathbf{4 d}\left(\mathrm{R}^{1}=\mathrm{R}^{2}=\mathrm{Me}\right)$ is commercially available.

(2) Sandrinelli, F.; Perrio, S.; Beslin, P. J. Org. Chem. 1997, 62, 8626-8627.

(3) (a) Davis, F. A.; Reddy, R. T.; Han, W.; Carroll, P. J. J. Am. Chem. Soc. 1992, 114, 1428-1437. (b) Davis, F. A., Weismiller, M. C., Murphy, C. K.; Reddy, R. T.; Chen, B.-C. J. Org. Chem. 1992, 57, 7274-7285. 
General Procedure for the Synthesis of $\beta$-Sulfanylesters 4. Ethyl acrylate ( $20 \mathrm{mmol}, 1$ eq) was added dropwise (exothermic reaction) to a suspension of thiol (20 mmol, 1 eq) and potassium carbonate $(1 \mathrm{mmol}, 0.05 \mathrm{eq})$ in $\mathrm{CH}_{2} \mathrm{Cl}_{2}(5 \mathrm{~mL})$ The resulting mixture was stirred at room temperature for $18 \mathrm{~h}$. After addition of $\mathrm{CH}_{2} \mathrm{Cl}_{2}(10 \mathrm{~mL})$, the reaction mixture was washed successively with aqueous $1 \mathrm{M} \mathrm{NaOH}$ solution $(15 \mathrm{~mL})$, water $(4 \times 15 \mathrm{~mL})$ until the $\mathrm{pH}$ is neutral, saturated aqueous $\mathrm{NaCl}$ solution $(15 \mathrm{~mL})$ and dried over $\mathrm{MgSO}_{4}$. Filtration and evaporation to dryness afforded the anticipated sulfide $\mathbf{4}$, which was used in the next step without further purification.

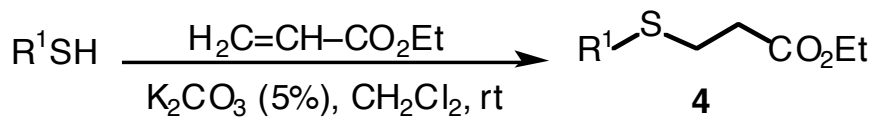

3-(p-Tolylsulfanyl)propionic acid ethyl ester $4 a^{4}{ }^{4}$ Obtained from 4-methylthiophenol $\left(\mathrm{R}^{1}=4-\mathrm{MeC}_{6} \mathrm{H}_{4}, 2.48 \mathrm{~g}, 20.0 \mathrm{mmol}\right)$. Yield 95\% (4.25 g, $\left.18.9 \mathrm{mmol}\right)$. Orange oil. TLC $\left(\mathrm{CH}_{2} \mathrm{Cl}_{2} /\right.$ pentane, 30:70) $R f=0.26 .{ }^{1} \mathrm{H}$ NMR $\left(400 \mathrm{MHz}, \mathrm{CDCl}_{3}\right): \delta 1.25(\mathrm{t}, J=7.1,3 \mathrm{H}), 2.32(\mathrm{~s}$, $3 \mathrm{H}), 2.58(\mathrm{t}, J=7.5,2 \mathrm{H}), 3.11(\mathrm{t}, J=7.5,2 \mathrm{H}), 4.12(\mathrm{q}, J=7.1,2 \mathrm{H}), 7.10\left(\mathrm{~d}_{\text {app }}, J=8.1,2 \mathrm{H}\right), 7.26$ $\left(\mathrm{d}_{\text {app }}, J=8.1,2 \mathrm{H}\right) .{ }^{13} \mathrm{C} \mathrm{NMR}\left(62.9 \mathrm{MHz}, \mathrm{CDCl}_{3}\right): \delta 14.6,21.4,30.2,35.0,61.1,130.2,131.5$, 131.8, 137.2, 171.9. IR $\left(\mathrm{NaCl}, \mathrm{cm}^{-1}\right): v 2978,2926,1734,1242,1178 . \mathrm{MS}$ (EI) $\mathrm{m} / z 224\left(\mathrm{M}^{+}\right.$, 100), 179 (13), 123 (17), 91 (15).

3-(2-Methoxyphenylsulfanyl)propionic acid ethyl ester $4 b .^{5}$ Obtained from 2-methoxythiophenol $\left(\mathrm{R}^{1}=2-\mathrm{MeOC}_{6} \mathrm{H}_{4}, 1.40 \mathrm{~g}, 10.0 \mathrm{mmol}\right)$. Yield 96\% (2.31g, $\left.9.6 \mathrm{mmol}\right)$. Pale yellow oil. TLC $\left(\mathrm{CH}_{2} \mathrm{Cl}_{2} /\right.$ pentane, $\left.40: 60\right) R f=0.51 .{ }^{1} \mathrm{H} \mathrm{NMR}\left(400 \mathrm{MHz}, \mathrm{CDCl}_{3}\right): \delta 1.25(\mathrm{t}, J=$ $7.1,3 \mathrm{H}), 2.61(\mathrm{t}, J=7.6,2 \mathrm{H}), 3.14(\mathrm{t}, J=7.6,2 \mathrm{H}), 3.89(\mathrm{~s}, 3 \mathrm{H}), 4.13(\mathrm{q}, J=7.1,2 \mathrm{H}), 6.86\left(\mathrm{~d}_{\text {app }}\right.$, $J=8.1,1 \mathrm{H}), 6.92(\mathrm{dt}, J=7.6, J=1.0,1 \mathrm{H}), 7.22(\mathrm{dt}, J=8.1, J=1.5,1 \mathrm{H}), 7.32(\mathrm{dd}, J=7.6, J=$

(4) Shi, D.; Lu, Z.; Mu, L.; Dai, G. Synth. Commun. 1998, 28, 1073-1078.

(5) Montanari, S.; Cavalleri, P.; Santangelo, F. Italy Patent WO 9507885, 1995. 
1.5, 1H). ${ }^{13} \mathrm{C} \mathrm{NMR}\left(62.9 \mathrm{MHz}, \mathrm{CDCl}_{3}\right): \delta 14.6,27.8,34.8,56.2,61.0,111.1,121.4,123.5,128.4$, 131.3, 158.3, 172.3. IR $\left(\mathrm{NaCl}, \mathrm{cm}^{-1}\right): v$ 3062, 2980, 1732, 1242. MS (EI) $\mathrm{m} / z 240\left(\mathrm{M}^{+}, 100\right), 212$ (1), 195 (10), 166 (20), 153 (14), 137 (11), 125 (5), 108 (9), 77 (5).

3-(2,6-Dimethylphenylsulfanyl)propionic acid ethyl ester 4c. Obtained from 2,6-dimethylthiophenol $\left(\mathrm{R}^{1}=2,6-\mathrm{Me}_{2} \mathrm{C}_{6} \mathrm{H}_{3}, 690 \mathrm{mg}, 4.99 \mathrm{mmol}\right)$. Yield 87\% (1.04 g, 4.36 mmol). Colorless oil. TLC $\left(\mathrm{CH}_{2} \mathrm{Cl}_{2} /\right.$ pentane, 20:80) $R f=0.14 .{ }^{1} \mathrm{H}$ NMR $\left(250 \mathrm{MHz}, \mathrm{CDCl}_{3}\right): \delta$ $1.23(\mathrm{t}, J=7.1,3 \mathrm{H}), 2.49(\mathrm{t}, J=7.4,2 \mathrm{H}), 2.53(\mathrm{~s}, 6 \mathrm{H}), 2.92(\mathrm{t}, J=7.4,2 \mathrm{H}), 4.09(\mathrm{q}, J=7.1,2 \mathrm{H})$, 7.10 (broad s, 3H). ${ }^{13} \mathrm{C}$ NMR $\left(62.9 \mathrm{MHz}, \mathrm{CDCl}_{3}\right): \delta$ 14.3, 22.1, 30.1, 35.0, 60.8, 128.3, 128.6, 132.7, 143.4, 172.0. IR $\left(\mathrm{NaCl}, \mathrm{cm}^{-1}\right): v$ 2978, 2930, 1734, 1242, 1178. MS (EI) m/z $238\left(\mathrm{M}^{+}\right.$, 100), 137 (33), 105 (32). HRMS (EI) calcd for $\mathrm{C}_{13} \mathrm{H}_{18} \mathrm{O}_{2} \mathrm{~S}\left(\mathrm{M}^{+}\right)$238.1028, found 238.1010.

3-(n-Butylsulfanyl)propionic acid ethyl ester $4 e^{6}{ }^{6}$ Obtained from $n$-butanethiol $\left(\mathrm{R}^{1}=n\right.$ $\mathrm{Bu}, 2.12 \mathrm{~mL}, 20.0 \mathrm{mmol})$. Yield $93 \%$ (3.54 g, $18.6 \mathrm{mmol})$. Colorless oil. TLC $\left(\mathrm{CH}_{2} \mathrm{Cl}_{2} /\right.$ pentane, 40:60) $R f=0.20 .{ }^{1} \mathrm{H} \mathrm{NMR}\left(400 \mathrm{MHz}, \mathrm{CDCl}_{3}\right): \delta 0.91(\mathrm{t}, J=7.3,3 \mathrm{H}), 1.27(\mathrm{t}, J=7.1,3 \mathrm{H}), 1.40$ $($ sext, $J=7.3,2 \mathrm{H}), 1.54$ (quint, $J=7.3,2 \mathrm{H}), 2.81(\mathrm{t}, J=7.3,2 \mathrm{H}), 4.16(\mathrm{q}, J=7.1,2 \mathrm{H}) .{ }^{13} \mathrm{C}$ NMR $\left(62.9 \mathrm{MHz}, \mathrm{CDCl}_{3}\right): \delta 13.6,14.2,21.9,26.9,31.6,31.8,34.9,60.5,171.9 . \mathrm{IR}\left(\mathrm{NaCl}, \mathrm{cm}^{-1}\right): v$ 2958, 2870, 1738, 1244, 1178. MS (EI) m/z 190 (M+, 100), 145 (19), 117 (37), 101 (64).

3-(Benzylsulfanyl)propionic acid ethyl ester $4 f^{7}$ Obtained from benzyl mercaptan $\left(\mathrm{R}^{1}=\right.$ Bn, $2.28 \mathrm{~mL}, 20.0 \mathrm{mmol})$. Yield 92\% (4.14 g, $18.5 \mathrm{mmol})$. Colorless oil. TLC $\left(\mathrm{CH}_{2} \mathrm{Cl}_{2} /\right.$ pentane, 30:70) $R f=0.19 .{ }^{1} \mathrm{H} \mathrm{NMR}\left(250 \mathrm{MHz}, \mathrm{CDCl}_{3}\right): \delta 1.25(\mathrm{t}, J=7.5,3 \mathrm{H}), 2.54(\mathrm{t}, J=8.2,2 \mathrm{H}), 2.66$ (t, $J=8.2,2 \mathrm{H}), 3.73(\mathrm{~s}, 2 \mathrm{H}), 4.13(\mathrm{q}, J=7.5,2 \mathrm{H}), 7.22-7.32(\mathrm{~m}, 5 \mathrm{H}) \cdot{ }^{13} \mathrm{C}$ NMR $(62.9 \mathrm{MHz}$,

(6) Derzhinskii, A. R.; Konyushkin, L. D.; Prilezhaeva, E. N. Izv. Akad. Nauk, Ser. Khim. 1978, 27, 2070-2076.

(7) Tamura, Y.; Annoura, H.; Fuji, M.; Ishibashi, H. Chem. Pharm. Bull. 1986, 34, 540-549. 
$\left.\mathrm{CDCl}_{3}\right): \delta 14.3,26.4,34.7,36.4,60.7,127.2,128.6,128.9,138.2,171.9 . \mathrm{IR}\left(\mathrm{NaCl}, \mathrm{cm}^{-1}\right):$ v 3060 3028, 2980, 2928, 1734, 1242, 1180. MS (EI) m/z 224 (M+, 37 ), 123 (29), 91 (100).

3-(Cyclohexylsulfanyl)propionic acid ethyl ester $4 \mathrm{~g} .{ }^{8}$ Obtained from cyclohexyl mercaptan $\left(\mathrm{R}^{1}=\right.$ cyclo- $\left.\mathrm{C}_{6} \mathrm{H}_{11}, 2.45 \mathrm{~mL}, 20.0 \mathrm{mmol}\right)$. Yield 93\% (4.01 g, $\left.18.5 \mathrm{mmol}\right)$. Colorless oil. TLC $\left(\mathrm{CH}_{2} \mathrm{Cl}_{2} /\right.$ pentane, 50:50) $R f=0.36 .{ }^{1} \mathrm{H} \mathrm{NMR}\left(400 \mathrm{MHz}, \mathrm{CDCl}_{3}\right): \delta 1.27(\mathrm{t}, J=7.2,3 \mathrm{H})$, $1.29-1.33(\mathrm{~m}, 5 \mathrm{H}), 1.76-1.77(\mathrm{~m}, 2 \mathrm{H}), 1.95-1.99(\mathrm{~m}, 2 \mathrm{H}), 2.58(\mathrm{t}, J=7.5,2 \mathrm{H}), 2.63-2.69(\mathrm{~m}$, $1 \mathrm{H}), 2.80(\mathrm{t}, J=7.5,2 \mathrm{H}), 4.18(\mathrm{q}, J=7.2,2 \mathrm{H}) .{ }^{13} \mathrm{C} \mathrm{NMR}\left(62.9 \mathrm{MHz}, \mathrm{CDCl}_{3}\right): \delta 14.2,25.3,25.8$, 26.1, 33.6, 35.4, 43.6, 60.6, 172.0. IR $\left(\mathrm{NaCl}, \mathrm{cm}^{-1}\right):$ v 2980, 2928, 2852, 1736, 1242, 1176. MS (EI) $\mathrm{m} / z 216\left(\mathrm{M}^{+}, 100\right), 115$ (36). HRMS (CI, isobutane) calcd for $\mathrm{C}_{11} \mathrm{H}_{21} \mathrm{O}_{3} \mathrm{~S}\left(\mathrm{MH}^{+}\right)$233.1211, found 233.1203 .

3-(t-Butylsulfanyl)propionic acid ethyl ester 4h. ${ }^{9}$ Obtained from $t$-butyl mercaptan $\left(\mathrm{R}^{1}=\right.$ $t$-Bu, $2.25 \mathrm{~mL}, 20.0 \mathrm{mmol})$. Yield $66 \%(2.52 \mathrm{~g}, 13.2 \mathrm{mmol})$. Pale yellow oil. TLC $\left(\mathrm{CH}_{2} \mathrm{Cl}_{2} /\right.$ pentane, 50:50) $R f=0.27 .{ }^{1} \mathrm{H} \mathrm{NMR}\left(400 \mathrm{MHz}, \mathrm{CDCl}_{3}\right): \delta 1.26(\mathrm{t}, J=7.2,3 \mathrm{H}), 1.33(\mathrm{~s}$, 9H), $2.56(\mathrm{t}, J=7.8,2 \mathrm{H}), 2.79(\mathrm{t}, J=7.8,2 \mathrm{H}), 4.16(\mathrm{q}, J=7.2,2 \mathrm{H}) .{ }^{13} \mathrm{C}$ NMR $(62.9 \mathrm{MHz}$, $\left.\mathrm{CDCl}_{3}\right): \delta 13.8,23.0,30.5,34.6,42.0,60.2,172.2 . \mathrm{IR}\left(\mathrm{NaCl}, \mathrm{cm}^{-1}\right): \vee 2962,2900,2864,1736$, 1242, 1164. MS (EI) m/z $190\left(\mathrm{M}^{+}, 52\right), 134$ (96), 101 (23), 57 (100).

(8) Brodin, R.; Boigegrain, R.; Bignon, E.; Molimard, J.-C.; Olliero, D. France Patent WO 9915525, 1999.

(9) Billard, T.; Roques, N.; Langlois, B. R. J. Org. Chem. 1999, 11, 3813-3820. 
General Procedure for the Synthesis of $\beta$-Sulfinylesters 1. A solution of $\beta$-sulfanylester $4(1 \mathrm{mmol}, 1 \mathrm{eq})$ in $\mathrm{MeOH}(10 \mathrm{~mL})$ was cooled to $0{ }^{\circ} \mathrm{C}$ and a solution of $\mathrm{NaIO}_{4}(1 \mathrm{mmol}, 1 \mathrm{eq})$ in water $(5 \mathrm{~mL})$ was added dropwise. The reaction mixture was stirred overnight allowing the cold bath warm slowly to room temperature. Liberated sodium iodate was filtered and methanol was removed under vacuum and the residue extracted with EtOAc $(2 \times 10 \mathrm{~mL})$. The combined organic layers were washed with saturated aqueous $\mathrm{NaCl}$ solution $(20 \mathrm{~mL})$, dried over $\mathrm{MgSO}_{4}$, filtered and evaporated to dryness. The crude product was then purified by column chromatography on silica gel to afford pure sulfinylesters $\mathbf{1}$.

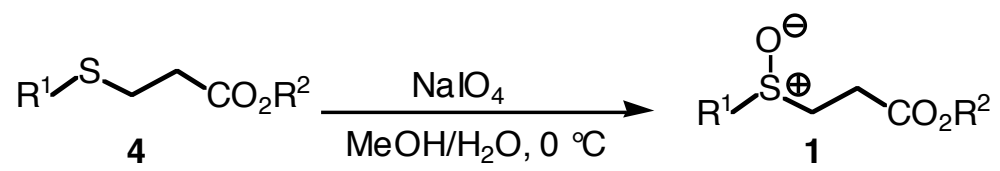

3-(p-Tolylsulfinyl)propionic acid ethyl ester 1a. Obtained from $\beta$-sulfanylester $4 a\left(R^{1}=\right.$ 4- $\left.\mathrm{MeC}_{6} \mathrm{H}_{4}, \mathrm{R}^{2}=\mathrm{Et}, 224 \mathrm{mg}, 1.00 \mathrm{mmol}\right)$. Yield 90\% (216 mg, $\left.0.90 \mathrm{mmol}\right)$. Colorless oil. TLC $\left(\mathrm{CH}_{2} \mathrm{Cl}_{2} / \mathrm{AcOEt}, 80: 20\right) R f=0.30 .{ }^{1} \mathrm{H}$ NMR $\left(400 \mathrm{MHz}, \mathrm{CDCl}_{3}\right): \delta 1.24(\mathrm{t}, J=7.2,3 \mathrm{H}), 2.42(\mathrm{~s}$, $3 \mathrm{H}), 2.53$ (ddd, $J=5.7,8.6$ and 17.1,1H), 2.79 (ddd, $J=6.6,8.5$ and 17.1, 1H), 2.95 (ddd, $J=$ $5.7,8.5$ and $13.4,1 \mathrm{H}), 3.18(\mathrm{ddd}, J=6.6,8.6$ and $13.4,1 \mathrm{H}), 4.11(\mathrm{q}, J=7.2,2 \mathrm{H}), 7.32\left(\mathrm{~d}_{\text {app }}, J=\right.$ 8.1, 2H), $7.50\left(\mathrm{~d}_{\text {app }}, J=8.1,2 \mathrm{H}\right) .{ }^{13} \mathrm{C} \mathrm{NMR}\left(62.9 \mathrm{MHz}, \mathrm{CDCl}_{3}\right): \delta 14.1,21.4,26.7,51.2,61.1$, 124.1, 130.0, 139.7, 141.7, 171.2. IR $\left(\mathrm{NaCl}, \mathrm{cm}^{-1}\right): v 2980,2926,1734,1236,1180,1046 . \mathrm{MS}$ (EI) m/z $240\left(\mathrm{M}^{+}, 43\right), 224$ (19), 195 (11), 140 (40), 139 (100), 123 (40), 92 (35), 91 (36), 77 (14), 65 (20). HRMS (EI) calcd for $\mathrm{C}_{12} \mathrm{H}_{16} \mathrm{O}_{3} \mathrm{~S}\left(\mathrm{M}^{+}\right)$240.0820, found 240.0818.

3-(2-Methoxyphenylsulfinyl)propionic acid ethyl ester 1b. Obtained from $\beta$-sulfanylester $\mathbf{4 b}\left(\mathrm{R}^{1}=2-\mathrm{MeOC}_{6} \mathrm{H}_{4}, \mathrm{R}^{2}=\mathrm{Et}, 2.31 \mathrm{~g}, 9.6 \mathrm{mmol}\right)$. Yield 98\% (2.42 g, $\left.9.5 \mathrm{mmol}\right)$. Yellow oil. TLC $\left(\mathrm{CH}_{2} \mathrm{Cl}_{2} / \mathrm{AcOEt}\right.$, 90:10) $R f=0.26 .{ }^{1} \mathrm{H} \mathrm{NMR}\left(400 \mathrm{MHz}, \mathrm{CDCl}_{3}\right): \delta 1.21(\mathrm{t}, J=$ 7.1, 3H), $2.47(\mathrm{ddd}, J=6.0,9.1$ and 17.1, 1H), $2.80(\mathrm{ddd}, J=6.0,9.1$ and 17.1, 1H), 3.12 (ddd, $J$ 
$=6.0,9.1$ and $15.1,1 \mathrm{H}), 3.36(\mathrm{ddd}, J=6.0,9.1$ and $15.1,1 \mathrm{H}), 3.88(\mathrm{~s}, 3 \mathrm{H}), 4.03-4.07(\mathrm{~m}, 2 \mathrm{H})$, $6.91\left(\mathrm{~d}_{\text {app }}, J=8.2,1 \mathrm{H}\right), 7.17(\mathrm{dt}, J=7.5$ and $0.8,1 \mathrm{H}), 7.43-7.47(\mathrm{~m}, 1 \mathrm{H}), 7.74(\mathrm{dd}, J=1.7$ and 7.5, 1H). ${ }^{13} \mathrm{C} \mathrm{NMR}\left(62.9 \mathrm{MHz}, \mathrm{CDCl}_{3}\right): \delta$ 14.5, 26.0, 47.0, 56.2, 61.3, 111.0, 121.9, 126.1, 130.1, 132.6, 155.5, 172.0. IR $\left(\mathrm{NaCl}, \mathrm{cm}^{-1}\right): v 3062,2980,1732,1274,1238,1036 . \mathrm{MS}(\mathrm{EI}) \mathrm{m} / \mathrm{z} 256$ (M+ $\left.\mathrm{M}^{+}, 40\right), 239$ (82), 211 (17), 155 (100), 139 (36), 125 (67), 108 (33), 101 (45), 97 (36), 84 (19), 77 (18), 73 (34), 65 (12). HRMS (EI) calcd for $\mathrm{C}_{12} \mathrm{H}_{16} \mathrm{O}_{4} \mathrm{~S}\left(\mathrm{M}^{+}\right)$256.0769, found 256.0776.

3-(2,6-Dimethylphenylsulfinyl)propionic acid ethyl ester 1c. Obtained from $\beta$-sulfanylester $4 \mathbf{c}\left(\mathrm{R}^{1}=2,6-\mathrm{Me}_{2} \mathrm{C}_{6} \mathrm{H}_{3}, \mathrm{R}^{2}=\mathrm{Et}, 892 \mathrm{mg}, 3.74 \mathrm{mmol}\right)$. Yield 69\% (652 mg, 2.57 mmol). Pale yellow oil. TLC $\left(\mathrm{CH}_{2} \mathrm{Cl}_{2}\right) R f=0.11 .{ }^{1} \mathrm{H} \mathrm{NMR}\left(400 \mathrm{MHz}, \mathrm{CDCl}_{3}\right): \delta 1.24(\mathrm{t}, J=7.1$, $3 \mathrm{H}), 2.56(\mathrm{~s}, 6 \mathrm{H}), 2.76-3.82(\mathrm{~m}, 2 \mathrm{H}), 3.11(\mathrm{dt}, J=13.2$ and $7.8,1 \mathrm{H}), 3.43(\mathrm{ddd}, J=6.2,6.9$ and 13.2, 1H), $4.12(\mathrm{q}, J=7.1,2 \mathrm{H}), 7.02(\mathrm{~d}, J=7.6,2 \mathrm{H}), 7.22(\mathrm{t}, J=7.6,1 \mathrm{H}) .{ }^{13} \mathrm{C} \mathrm{NMR}(62.9 \mathrm{MHz}$, $\left.\mathrm{CDCl}_{3}\right): \delta 14.2,19.2,28.5,47.2,61.3,130.4,131.1,137.8,138.5,171.2 . \mathrm{IR}\left(\mathrm{NaCl}, \mathrm{cm}^{-1}\right): v 3056$, 2980, 2932, 1729, 1212, 1186, 1060. MS (CI, isobutane) $\mathrm{m} / z 293\left[\left(\mathrm{M}+\mathrm{C}_{3} \mathrm{H}_{3}{ }^{+}\right), 6\right], 256(17), 255$ $\left(\mathrm{MH}^{+}, 100\right)$. HRMS (CI, isobutane) calcd for $\mathrm{C}_{13} \mathrm{H}_{19} \mathrm{O}_{3} \mathrm{~S}\left(\mathrm{MH}^{+}\right)$255.1055, found 255.1060.

3-(Methylsulfinyl)propionic acid methyl ester $1 d$. Obtained from $\beta$-sulfanylester $4 d\left(R^{1}\right.$ $\left.=\mathrm{Me}, \mathrm{R}^{2}=\mathrm{Me}, 627 \mu \mathrm{L}, 5.00 \mathrm{mmol}\right) . \mathrm{A} 41 \%$ yield $(338 \mathrm{mg}, 2.06 \mathrm{mmol})$ was obtained with the general procedure described above. Improvement has been achieved using an anhydrous oxidation protocol described by Camps. ${ }^{10}$ To freshly activated KF $\left(3 \mathrm{~h}\right.$ at $120{ }^{\circ} \mathrm{C}$ under reduced pressure, $174 \mathrm{mg}, 3 \mathrm{mmol}, 1.5$ eq.) was added a solution of $m$-CPBA (492 mg, $2 \mathrm{mmol}, 2$ eq.) in $\mathrm{CH}_{2} \mathrm{Cl}_{2}(8 \mathrm{~mL})$ previously dried over $\mathrm{MgSO}_{4}$. The reaction mixture was cooled to $0{ }^{\circ} \mathrm{C}$ and a solution of 3-(methylsulfanyl)propionic acid methyl ester $\mathbf{4 d}\left(251 \mu \mathrm{L}, 2 \mathrm{mmol}, 1\right.$ eq.) in $\mathrm{CH}_{2} \mathrm{Cl}_{2}$ $(4 \mathrm{~mL})$ was added dropwise. The reaction mixture was stirred at $0{ }^{\circ} \mathrm{C}$ for $3 \mathrm{~h}$ and at room

(10) Camps, F.; Coll, J.; Messeguer, A.; Pujol, F. J. Org. Chem. 1982, 47, 5402-5404. 
temperature overnight. The suspension was filtered over celite and the solvent was removed under reduced pressure. The crude product was purified by column chromatography eluting with ethyl acetate. Yield 79\% (256 mg, $1.57 \mathrm{mmol})$. Colorless oil. TLC (AcOEt/ $\left.\mathrm{CH}_{2} \mathrm{Cl}_{2}, 40: 60\right) R f=$ 0.08. ${ }^{1} \mathrm{H}$ NMR $\left(250 \mathrm{MHz}, \mathrm{CDCl}_{3}\right): \delta 2.61(\mathrm{~s}, 3 \mathrm{H}), 2.82-3.08(\mathrm{~m}, 4 \mathrm{H}), 3.74(\mathrm{~s}, 3 \mathrm{H}) .{ }^{13} \mathrm{C}$ NMR $\left(62.9 \mathrm{MHz}, \mathrm{CDCl}_{3}\right): \delta 26.9,39.0,49.1,52.4,171.9 . \mathrm{IR}\left(\mathrm{NaCl}, \mathrm{cm}^{-1}\right): v$ 3001, 2955, 2920, 1731 , 1439, 1365, 1303, 1247, 1028. MS (EI) m/z $151\left(\mathrm{MH}^{+}, 30\right), 150\left(\mathrm{M}^{+}, 39\right), 135$ (42), 119 (34), 103 (16), 87 (100), 79 (17), 64 (47), 63 (99), 61 (21). HRMS (EI) calcd for $\mathrm{C}_{5} \mathrm{H}_{10} \mathrm{O}_{3} \mathrm{~S}\left(\mathrm{M}^{+}\right)$150.0351, found 150.0342 .

3-(n-Butylsulfinyl)propionic acid ethyl ester 1e. Obtained from $\beta$-sulfanylester $4 \mathrm{e}\left(\mathrm{R}^{1}=\right.$ $n$-Bu, R² = Et, $2.91 \mathrm{~g}, 15.3 \mathrm{mmol})$. Yield 96\% (3.03 g, $14.7 \mathrm{mmol})$. Colorless oil. TLC $\left(\mathrm{CH}_{2} \mathrm{Cl}_{2} / \mathrm{AcOEt}, 90: 10\right) R f=0.21 .{ }^{1} \mathrm{H} \mathrm{NMR}\left(400 \mathrm{MHz}, \mathrm{CDCl}_{3}\right): \delta 0.93(\mathrm{t}, J=7.4,3 \mathrm{H}), 1.26(\mathrm{t}, J$ $=7.1,3 \mathrm{H}), 1.40-1.55(\mathrm{~m}, 2 \mathrm{H}), 1.70-1.79(\mathrm{~m}, 2 \mathrm{H}), 2.62-2.88(\mathrm{~m}, 5 \mathrm{H}), 2.96-3.04(\mathrm{~m}, 1 \mathrm{H}), 4.16$ $(\mathrm{q}, J=7.1,2 \mathrm{H}) .{ }^{13} \mathrm{C} \mathrm{NMR}\left(62.9 \mathrm{MHz}, \mathrm{CDCl}_{3}\right): \delta 13.8,14.2,22.2,24.7,27.3,47.0,52.6,61.3$, 171.6. IR $\left(\mathrm{NaCl}, \mathrm{cm}^{-1}\right): v 2960,2871,1734,1374,1184,1028 . \mathrm{MS}$ (CI, isobutane) $\mathrm{m} / z 245$ $\left[\left(\mathrm{M}+\mathrm{C}_{3} \mathrm{H}_{3}^{+}\right), 4\right], 208(12), 207\left(\mathrm{MH}^{+}, 100\right)$. HRMS (CI, isobutane) calcd for $\mathrm{C}_{9} \mathrm{H}_{19} \mathrm{O}_{3} \mathrm{~S}\left(\mathrm{MH}^{+}\right)$ 207.1055, found 207.1058.

3-(Benzylsulfinyl)propionic acid ethyl ester 1f. ${ }^{11}$ Obtained from $\beta$-sulfanylester $4 \mathbf{f}\left(\mathrm{R}^{1}=\right.$ $\mathrm{Bn}, \mathrm{R}^{2}=\mathrm{Et}, 224 \mathrm{mg}, 1.00 \mathrm{mmol}$ ). Yield $99 \%$ (238 mg, $\left.0.99 \mathrm{mmol}\right)$. White solid. mp 64-65 ${ }^{\circ} \mathrm{C}$ (Litt.: $\left.{ }^{11} 62-63{ }^{\circ} \mathrm{C}\right)$. TLC $\left(\mathrm{CH}_{2} \mathrm{Cl}_{2} / \mathrm{AcOEt}, 90: 10\right) R f=0.15 .{ }^{1} \mathrm{H}$ NMR $\left(400 \mathrm{MHz}, \mathrm{CDCl}_{3}\right): \delta 1.26$ $(\mathrm{t}, J=7.2,3 \mathrm{H}), 2.74-2.98(\mathrm{~m}, 4 \mathrm{H}), 3.99$ and $4.06(\mathrm{AB}, J=12.9,2 \mathrm{H}), 4.16(\mathrm{q}, J=7.2,2 \mathrm{H})$ 7.30-7.40 (m, 5H). ${ }^{13} \mathrm{C}$ NMR (62.9 $\left.\mathrm{MHz} \mathrm{CDCl}_{3}\right): \delta 14.2,27.0,45.6,58.7,61.2,128.6$,

(11) Wolfe S. et al. Can. J. Chem. 1979, 57, 2412-2425. 
129.1, 129.7, 130.1, 171.4. IR $\left(\mathrm{KBr}, \mathrm{cm}^{-1}\right): v$ 3084, 3060, 3028, 2966, 2914, 1722, 1246, 1184. MS (EI) m/z $240\left(\mathrm{M}^{+}, 2\right), 224$ (2), 195 (1), 123 (4), 92 (9), 91 (100), 77 (2), 65 (10).

3-(Cyclohexylsulfinyl)propionic acid ethyl ester 1g. Obtained from $\beta$-sulfanylester $\mathbf{4 g}$ $\left(\mathrm{R}^{1}=\right.$ cyclo- $\left.\mathrm{C}_{6} \mathrm{H}_{11}, \mathrm{R}^{2}=\mathrm{Et}, 4.00 \mathrm{~g}, 18.5 \mathrm{mmol}\right)$. Yield 84\% (3.60 g, $\left.15.5 \mathrm{mmol}\right)$. Colorless oil. TLC $\left(\mathrm{CH}_{2} \mathrm{Cl}_{2}\right) R f=0.19 .{ }^{1} \mathrm{H} \mathrm{NMR}\left(400 \mathrm{MHz}, \mathrm{CDCl}_{3}\right): \delta 1.27(\mathrm{t}, J=7.1,3 \mathrm{H}), 1.30-2.16(\mathrm{~m}$, $10 \mathrm{H}), 2.54-2.59(\mathrm{~m}, 1 \mathrm{H}), 2.80-3.04(\mathrm{~m}, 4 \mathrm{H}), 4.18(\mathrm{q}, J=7.1,2 \mathrm{H}) .{ }^{13} \mathrm{C} \mathrm{NMR}\left(62.9 \mathrm{MHz}, \mathrm{CDCl}_{3}\right)$ :

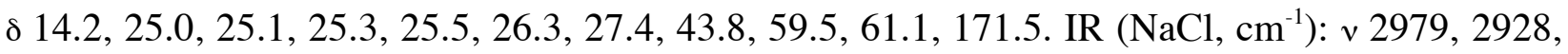
2852, 1736, 1242, 1177. MS (CI, isobutane) $m / z 271\left[\left(\mathrm{M}+\mathrm{C}_{3} \mathrm{H}_{3}{ }^{+}\right), 4\right], 233\left(\mathrm{MH}^{+}, 100\right)$. HRMS (CI, isobutane) calcd for $\mathrm{C}_{11} \mathrm{H}_{21} \mathrm{O}_{3} \mathrm{~S}\left(\mathrm{MH}^{+}\right)$233.1211, found 233.1203.

3-(t-Butylsulfinyl)propionic acid ethyl ester $1 \mathrm{~h} .{ }^{12}$ Obtained from $\beta$-sulfanylester $4 \mathrm{~h}\left(\mathrm{R}^{1}\right.$ $=t$-Bu, $\left.\mathrm{R}^{2}=\mathrm{Et}, 2.52 \mathrm{~g}, 13.2 \mathrm{mmol}\right)$. Yield $70 \%(1.92 \mathrm{~g}, 9.3 \mathrm{mmol})$. Colorless oil. TLC (AcOEt/pentane, 50:50) $R f=0.14 .{ }^{1} \mathrm{H} \mathrm{NMR}\left(250 \mathrm{MHz}, \mathrm{CDCl}_{3}\right)$ : $\delta 1.27(\mathrm{~s}, 9 \mathrm{H}), 1.27(\mathrm{t}, J=7.1$, 3H), 2.64-2.90 (m, 4H), 4.18 (q, $J=7.1,2 \mathrm{H}) .{ }^{13} \mathrm{C} \mathrm{NMR}\left(62.9 \mathrm{MHz}, \mathrm{CDCl}_{3}\right): \delta 14.3,22.9,28.3$, 40.8, 57.7, 61.1, 171.6. IR $\left(\mathrm{NaCl}, \mathrm{cm}^{-1}\right):$ v 2976, 2938, 2906, 2872, 1734, 1234, 1182, 1038. MS (EI) $m / z 207\left(\mathrm{MH}^{+}, 34\right), 150$ (59), 104 (100), 57 (82).

(12) Shelton, J. R.; Davis, K. E. Int. J. Sulfur Chem. 1973, 8, 205-216. 


\section{Typical Procedure for the Preparation of Racemic Sulfoxides 2 with $t$-BuOK as}

Base. A solution of $\beta$-sulfinylester $1(1.0 \mathrm{mmol}, 1 \mathrm{eq})$ in dry THF $(5 \mathrm{~mL})$ was cooled to $-78{ }^{\circ} \mathrm{C}$ and $t$-BuOK (1.1 mL of a 1M solution in THF, $1.1 \mathrm{mmol}, 1.1 \mathrm{eq})$ was added. After stirring at -78 ${ }^{\circ} \mathrm{C}$ for $20 \mathrm{~min}$, the alkyl halide ( $\left.1.2 \mathrm{mmol}, 1.2 \mathrm{eq}\right)$ was added. The reaction mixture was stirred at $-78^{\circ} \mathrm{C}$ for $1 \mathrm{~h} 30$ and then allowed to warm to room temperature for $18 \mathrm{~h}$. After concentration in vacuum, EtOAc $(15 \mathrm{~mL})$ was added and the organic layer was washed with water $(15 \mathrm{~mL})$. The aqueous layer was extracted with EtOAc $(3 \times 15 \mathrm{~mL})$. The organic layers were combined, washed with saturated aqueous $\mathrm{NaCl}(50 \mathrm{~mL})$, dried over $\mathrm{MgSO}_{4}$, filtered and evaporated to dryness. The resulting crude product was then purified by column chromatography to afford anticipated racemic sulfoxide 2 . Using methyl or ethyl iodides as the electrophile, sulfenate ester 3 (O-alkylation product) was also formed and readily separated from the major sulfoxide (S-alkylation products).

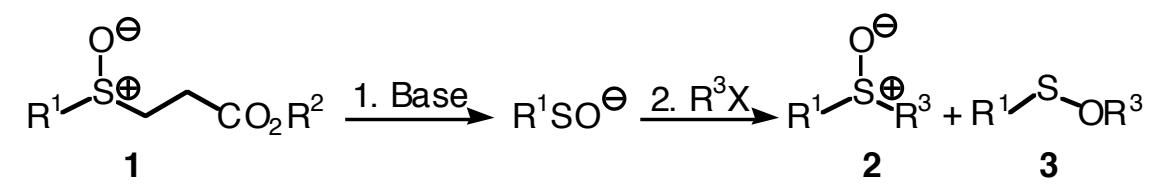

1-Benzylsulfinyl-4-methylbenzene $2 \mathbf{a}_{1}{ }^{13}$ Obtained from $\beta$-sulfinylester $\mathbf{1 a}\left(\mathrm{R}^{1}=\right.$ 4- $\mathrm{MeC}_{6} \mathrm{H}_{4}, \mathrm{R}^{2}=\mathrm{Et}, 240 \mathrm{mg}, 1.00 \mathrm{mmol}$ ) with benzyl bromide as the electrophile. Yield 77\% (176 mg, 0.77 mmol). White solid. mp 139-140 ${ }^{\circ} \mathrm{C}$ (Litt.: $\left.{ }^{13} 140-141{ }^{\circ} \mathrm{C}\right)$. TLC $\left(\mathrm{CH}_{2} \mathrm{Cl}_{2} / \mathrm{AcOEt}, 80: 20\right)$ $R f=0.43 .{ }^{1} \mathrm{H} \mathrm{NMR}\left(250 \mathrm{MHz}, \mathrm{CDCl}_{3}\right): \delta 2.39(\mathrm{~s}, 3 \mathrm{H}), 3.97$ and $4.09(\mathrm{AB}, J=12.5,2 \mathrm{H})$, 6.97-7.01 (m, 2H), 7.20-7.89 (m, 7H). ${ }^{13} \mathrm{C}$ NMR $\left(62.9 \mathrm{MHz}, \mathrm{CDCl}_{3}\right):$ ᄋ 21.4, 63.7, 124.5, 128.2, 128.4, 129.4, 129.6, 130.4, 139.7, 141.6. IR $\left(\mathrm{KBr}, \mathrm{cm}^{-1}\right):$ v 3058, 3032, 2960, 2910, 1033. MS (CI, isobutane) $\mathrm{m} / z 271\left[\left(\mathrm{M}^{+} \mathrm{C}_{3} \mathrm{H}_{5}^{+}\right), 2\right], 269\left[\left(\mathrm{M}^{+} \mathrm{C}_{3} \mathrm{H}_{3}{ }^{+}\right), 2\right], 231\left(\mathrm{MH}^{+}, 100\right), 215(6)$.

(13) Kise, M.; Oae, S. Bull. Chem. Soc. Jpn. 1970, 43, 1426-1430. 
1-Methylsulfinyl-4-methylbenzene $2 \mathbf{a}_{2} \cdot{ }^{14}$ Obtained as the major alkylation product from $\beta$-sulfinylester 1a $\left(\mathrm{R}^{1}=4-\mathrm{MeC}_{6} \mathrm{H}_{4}, \mathrm{R}^{2}=\mathrm{Et}, 245 \mathrm{mg}, 1.02 \mathrm{mmol}\right)$ and methyl iodide as the electrophile. Yield $83 \%$ (131 mg, $0.85 \mathrm{mmol}$ ). White solid. mp: $43-44^{\circ} \mathrm{C}$, cyclohexane (Litt.: ${ }^{13}$ $\left.42-43^{\circ} \mathrm{C}\right)$. TLC (heptane/AcOEt, 50:50) $R f=0.08 .{ }^{1} \mathrm{H}$ NMR $\left(250 \mathrm{MHz}, \mathrm{CDCl}_{3}\right): \delta 2.42(\mathrm{~s}, 3 \mathrm{H})$, $2.70(\mathrm{~s}, 3 \mathrm{H}), 7.26-7.31(\mathrm{~m}, 2 \mathrm{H}), 7.52-7.56(\mathrm{~m}, 2 \mathrm{H}) .{ }^{13} \mathrm{C} \mathrm{NMR}\left(62.9 \mathrm{MHz}, \mathrm{CDCl}_{3}\right): \delta$ 21.8, 44.4, 123.9, 130.4, 141.9, 142.9. IR (KBr, cm $\left.{ }^{-1}\right): v 2928,1592,1490,1046 . \mathrm{MS}(\mathrm{EI}) \mathrm{m} / z 154\left(\mathrm{M}^{+}, 99\right)$, 139 (100), 138 (42), 91 (29), 77 (46), 65 (27), 63 (19). The corresponding sulfenic ester $\mathbf{3} \mathbf{a}_{2}$ resulting of the competing $O$-alkylation of the intermediate sulfenate was also produced. 4-Methylbenzenesulfenic acid methyl ester $3 \mathbf{a}_{2} \cdot{ }^{15}$ Yield $15 \%$ (23 mg, 0,15 mmol). Colorless oil. $\operatorname{TLC}\left(\mathrm{CH}_{2} \mathrm{Cl}_{2}\right) R f=0.32 .{ }^{1} \mathrm{H}$ NMR $\left(250 \mathrm{MHz}, \mathrm{CDCl}_{3}\right): \delta 2.44(\mathrm{~s}, 3 \mathrm{H}), 3.03(\mathrm{~s}, 3 \mathrm{H}), 7.35\left(\mathrm{~d}_{\text {app }}, J=\right.$ 8.0 Hz, 2H), 7.80-7.83 (m, 2H). ${ }^{13} \mathrm{C}$ NMR (62.9 MHz, $\left.\mathrm{CDCl}_{3}\right): \delta 21.8,44.7,127.5,130.1,137.9$, 144.8. IR $\left(\mathrm{NaCl}, \mathrm{cm}^{-1}\right): v 3010,2959,2926,2854,1320,1299,1287,1144,1090 . \mathrm{MS}(\mathrm{EI}) \mathrm{m} / \mathrm{z}$ $155\left(\mathrm{MH}^{+}, 86\right), 154\left(\mathrm{M}^{+}, 33\right), 139$ (11), 107 (75), 91 (100), 77 (4), 65 (28).

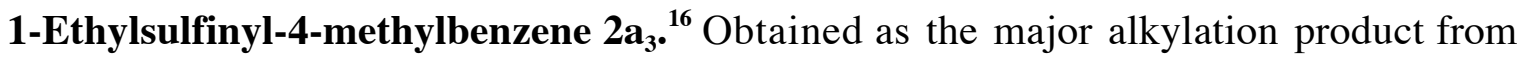
$\beta$-sulfinylester 1a $\left(\mathrm{R}^{1}=4-\mathrm{MeC}_{6} \mathrm{H}_{4}, \mathrm{R}^{2}=\mathrm{Et}, 224 \mathrm{mg}, 0.93 \mathrm{mmol}\right)$ and ethyl iodide as the electrophile. Yield $88 \%$ (138 mg, $0.82 \mathrm{mmol})$. Yellow oil. TLC $\left(\mathrm{CH}_{2} \mathrm{Cl}_{2}\right) R f=0.09 .{ }^{1} \mathrm{H}$ NMR $\left(400 \mathrm{MHz}, \mathrm{CDCl}_{3}\right): \delta 1.19(\mathrm{t}, J=7.5,3 \mathrm{H}), 2.42(\mathrm{~s}, 3 \mathrm{H}), 3.22$ and $3.28\left(\mathrm{AB}\right.$ part of $\mathrm{ABX}_{3}, J_{A B}=$ 13.2, $\left.J_{A X}=J_{B X}=7.5,2 \mathrm{H}\right), 7.32\left(\mathrm{~d}_{\mathrm{app}}, J=8.2,2 \mathrm{H}\right), 7.50\left(\mathrm{~d}_{\mathrm{app}}, J=8.2,2 \mathrm{H}\right) .{ }^{13} \mathrm{C} \mathrm{NMR}(62.9 \mathrm{MHz}$, $\left.\mathrm{CDCl}_{3}\right): \delta 6.0,21.4,50.4,124.2,129.8,140.1,141.4 . \mathrm{IR}\left(\mathrm{NaCl}, \mathrm{cm}^{-1}\right): v 3036,2976,2932,2872$, 1046. MS (EI) m/z $169\left(\mathrm{MH}^{+}, 55\right), 152$ (25), 140 (100), 92 (45). The corresponding sulfenic ester

(14) Kim, S. S.; Nehru, K.; Kim, S. S.; Kim, D. W.; Jung, H. C. Synthesis 2002, 17, 2484-2486. (15) Yoshimura, T.; Hamada, K.; Yamazaki, S.; Shimasaki, C.; Ono, S.; Tsukurimishi, E. Bull. Chem. Soc. Jpn. 1995, 68, 211-218.

(16) Mohraz, M.; Jian-qi, W.; Heilbronner, E.; Solladié-Cavallo, A.; Matloubi-Moghadam, F. Helv. Chim. Acta 1981, 64, 97-112. 
$\mathbf{3 \mathbf { a } _ { 3 }}$ resulting of the competing $O$-alkylation of the intermediate sulfenate was also produced. 4-Methylbenzenesulfenic acid ethyl ester $\mathbf{3 a}_{3} \cdot{ }^{17}$ Yield $5 \%$ (8 mg, $\left.0.05 \mathrm{mmol}\right)$. Orange oil, TLC $\left(\mathrm{CH}_{2} \mathrm{Cl}_{2}\right) R f=0.46 .{ }^{1} \mathrm{H}$ NMR $\left(250 \mathrm{MHz}, \mathrm{CDCl}_{3}\right): \delta 0.83-0.88(\mathrm{~m}, 3 \mathrm{H}), 2.46(\mathrm{~s}, 3 \mathrm{H}), 3.10(\mathrm{q}, J=$ 7.4, 2H), $7.36\left(\mathrm{~d}_{\text {app }}, J=8.2,2 \mathrm{H}\right), 7.79\left(\mathrm{~d}_{\text {app }}, J=8.2,2 \mathrm{H}\right) .{ }^{13} \mathrm{C} \mathrm{NMR}\left(62.9 \mathrm{MHz}, \mathrm{CDCl}_{3}\right): \delta 7.6$, $21.8,50.8,128.4,130.0,135.8,144.7 . \mathrm{IR}\left(\mathrm{NaCl}, \mathrm{cm}^{-1}\right): v 2924,2853,1313,1302,1274,1142$, 1086.

1-Methylsulfinyl-2-methoxybenzene $2 \mathrm{~b} .{ }^{18}$ Obtained as the major alkylation product from $\beta$-sulfinylester $\mathbf{1 b}\left(\mathrm{R}^{1}=2-\mathrm{MeOC}_{6} \mathrm{H}_{4}, \mathrm{R}^{2}=\mathrm{Et}, 256 \mathrm{mg}, 1.00 \mathrm{mmol}\right)$ and methyl iodide as the electrophile. Yield $80 \%$ (136 mg, $0.80 \mathrm{mmol})$. Pale yellow oil. TLC $\left(\mathrm{CH}_{2} \mathrm{Cl}_{2} / \mathrm{AcOEt}, 90: 10\right) R f=$ 0.18. ${ }^{1} \mathrm{H}$ NMR $\left(250 \mathrm{MHz}, \mathrm{CDCl}_{3}\right): \delta 2.78(\mathrm{~s}, 3 \mathrm{H}), 3.89(\mathrm{~s}, 3 \mathrm{H}), 6.92\left(\mathrm{~d}_{\text {app }}, J=8.2,1 \mathrm{H}\right), 7.21(\mathrm{dt}, J$ $=0.9$ and $7.5,1 \mathrm{H}), 7.44(\mathrm{dt}, J=1.7$ and $7.8,1 \mathrm{H}), 7.83(\mathrm{dd}, J=1.7$ and 7.8$) .{ }^{13} \mathrm{C}$ NMR $(62.9$ $\left.\mathrm{MHz}, \mathrm{CDCl}_{3}\right): \delta 41.3,55.7,110.6,121.7,124.7,131.9,133.2,154.8 . \mathrm{IR}\left(\mathrm{NaCl}, \mathrm{cm}^{-1}\right): v 3012$, 2941, 2840, 1477, 1274, 1067, 1021. MS (EI) $m / z 171\left(\mathrm{MH}^{+}, 69\right), 153$ (100), 125 (11). The corresponding sulfenic ester $\mathbf{3 b}$ resulting of the competing $O$-alkylation of the intermediate sulfenate was also produced. 2-Methoxybenzenesulfenic acid methyl ester 3b. Yield 18\% (31 mg, 0.18 mmol). White solid. mp: $90-91{ }^{\circ} \mathrm{C}, \mathrm{CH}_{2} \mathrm{Cl}_{2} /$ pentane. TLC $\left(\mathrm{CH}_{2} \mathrm{Cl}_{2}\right) R f=0.18 .{ }^{1} \mathrm{H} \mathrm{NMR}$ $\left(400 \mathrm{MHz}, \mathrm{CDCl}_{3}\right): \delta 3.22(\mathrm{~s}, 3 \mathrm{H}), 4.01(\mathrm{~s}, 3 \mathrm{H}), 7.05\left(\mathrm{~d}_{\mathrm{app}}, J=8.3,1 \mathrm{H}\right), 7.11\left(\mathrm{t}_{\mathrm{app}}, J=7.5,1 \mathrm{H}\right)$, 7.58-7.62 (m, 1H), $7.98\left(\mathrm{~d}_{\text {app }}, J=7.8,1 \mathrm{H}\right) \cdot{ }^{13} \mathrm{C} \mathrm{NMR}\left(62.9 \mathrm{MHz}, \mathrm{CDCl}_{3}\right): \delta 43.1,56.5,112.5$, 120.9, 128.6, 129.9, 135.7, 157.4. IR $\left(\mathrm{NaCl}, \mathrm{cm}^{-1}\right): v 2999,2953,2925,2852,1590,1481,1288$, 1132.

(17) Abbott, D. J.; Stirling, C. J. M. J. Chem. Soc. C 1969, 818-821.

(18) Benassi, R.; Folli, U.; Iarossi, D.; Mucci, A.; Schenetti, L.; Taddei, F. J. Chem. Soc. Perkin Trans. 2 1989, 517-522. 
1-Benzylsulfinyl-2,6-dimethylbenzene 2c. Obtained from $\beta$-sulfinylester $1 c\left(R^{1}=2,6\right.$ $\left.\mathrm{Me}_{2} \mathrm{C}_{6} \mathrm{H}_{3}, \mathrm{R}^{2}=\mathrm{Et}, 254 \mathrm{mg}, 1.00 \mathrm{mmol}\right)$ and benzyl bromide as the electrophile. Yield 68\% (165 $\mathrm{mg}, 0.68 \mathrm{mmol})$. White solid. Mp: 82-84 ${ }^{\circ} \mathrm{C} . \mathrm{TLC}\left(\mathrm{CH}_{2} \mathrm{Cl}_{2}\right) R f=0.39 .{ }^{1} \mathrm{H}$ NMR $(250 \mathrm{MHz}$, $\left.\mathrm{CDCl}_{3}\right)$ : $\delta 2.34$ (broad s, 6H), 4.37 (s, 2H), 6.96-7.04 (m, 4H), 7.18-7.28 (m, 4H). ${ }^{13} \mathrm{C}$ NMR $(62.9$ $\left.\mathrm{MHz}, \mathrm{CDCl}_{3}\right): \delta 19.1,58.7,128.2,128.6,130.0,130.1,130.3,131.0,136.8,139.1 . \mathrm{IR}\left(\mathrm{NaCl}, \mathrm{cm}^{-}\right.$ $\left.{ }^{1}\right): v 3057,2925,1729,1456,1055 . \mathrm{MS}(\mathrm{EI}) \mathrm{m} / \mathrm{z} 244\left(\mathrm{M}^{+}, 4\right), 228$ (1), 196 (2), 92 (11), 91 (100), 77 (6), 65 (15). HRMS (EI) calcd for $\mathrm{C}_{15} \mathrm{H}_{16} \mathrm{OS}\left(\mathrm{M}^{+}\right)$244.0922, found 244.0922.

[(Methylsulfinyl)methyl]benzene 2d. ${ }^{14}$ Obtained from $\beta$-sulfinylester $\mathbf{1 d}\left(\mathrm{R}^{1}=\mathrm{Me}, \mathrm{R}^{2}=\right.$ Me, $74 \mathrm{mg}, 0.50 \mathrm{mmol}$ ) and benzyl bromide as the electrophile. Yield 68\% (32 mg, $0.21 \mathrm{mmol}$ ). Pale yellow oil. TLC $\left(\mathrm{CH}_{2} \mathrm{Cl}_{2} / \mathrm{AcOEt}, 60: 40\right) R f=0.21 .{ }^{1} \mathrm{H} \mathrm{NMR}\left(250 \mathrm{MHz}, \mathrm{CDCl}_{3}\right): \delta 2.46(\mathrm{~s}$, $3 \mathrm{H}), 3.93$ and $4.07(\mathrm{AB}, J=12.8,2 \mathrm{H}), 7.26-7.39(\mathrm{~m}, 5 \mathrm{H}) .{ }^{13} \mathrm{C} \mathrm{NMR}\left(62.9 \mathrm{MHz}, \mathrm{CDCl}_{3}\right): \delta 37.4$, 60.4, 128.6, 129.1, 129.8, 130.1. IR $\left(\mathrm{NaCl}, \mathrm{cm}^{-1}\right): v 1724,1454,1026 . \mathrm{MS}(\mathrm{EI}) \mathrm{m} / z .154\left(\mathrm{M}^{+}, 3\right)$, 137 (4), 91 (100), 77 (4), 65 (13).

[(n-Butylsulfinyl)methyl]benzene $2 \mathrm{e}^{19}$ Obtained from $\beta$-sulfinylester $\mathbf{1 e}\left(\mathrm{R}^{1}=n\right.$-Bu, $\mathrm{R}^{2}$ = Et, $206 \mathrm{mg}, 1.00 \mathrm{mmol}$ ) and benzyl bromide as the electrophile. Yield 95\% (187 mg, 0.95 mmol). Pale yellow solid. mp $61-63{ }^{\circ} \mathrm{C}\left(\mathrm{Litt} .:^{19} 62{ }^{\circ} \mathrm{C}\right.$, benzene/Et $\left.2 \mathrm{O}\right)$. TLC $\left(\mathrm{CH}_{2} \mathrm{Cl}_{2} /\right.$ pentane, 80:20) $R f=0.42 .{ }^{1} \mathrm{H}$ NMR $\left(400 \mathrm{MHz}, \mathrm{CDCl}_{3}\right): \delta 0.91(\mathrm{t}, J=7.3,3 \mathrm{H}), 1.22-1.27(\mathrm{~m}, 1 \mathrm{H}), 1.35-$ $1.49(\mathrm{~m}, 2 \mathrm{H}), 1.66-1.79(\mathrm{~m}, 2 \mathrm{H}), 2.56(\mathrm{t}, J=7.8,2 \mathrm{H}), 3.93$ and $4.01(\mathrm{AB}, J=12.9,2 \mathrm{H})$, 7.26-7.39 (m, 5H). ${ }^{13} \mathrm{C}$ NMR (62.9 MHz, $\left.\mathrm{CDCl}_{3}\right): \delta$ 13.8, 22.1, 24.6, 50.8, 58.5, 60.5, 128.4, 129.1, 130.1, 130.2. IR $\left(\mathrm{KBr}, \mathrm{cm}^{-1}\right)$ : v 2980, 2936, 2872, 1731, 1631, 1446, 1371, 1260, 1180 , 1029. MS (EI) m/z 196 (M+, 2), 180 (1), 92 (10), 91 (100), 77 (1), 65 (14).

(19) Rajanikanth, B.; Ravindranath, B. Indian J. Chem. Sect. B 1984, 23, 877-878. 
[(Benzylsulfinyl)methyl]benzene 2f. ${ }^{19}$ Obtained from $\beta$-sulfinylester $\mathbf{1 f}\left(\mathrm{R}^{1}=\mathrm{Bn}, \mathrm{R}^{2}=\right.$ Et, $240 \mathrm{mg}, 1.00 \mathrm{mmol}$ ) and benzyl bromide as the electrophile. Yield 57\% (132 mg, $0.57 \mathrm{mmol})$. White solid. mp: $129-131{ }^{\circ} \mathrm{C}, \mathrm{CH}_{2} \mathrm{Cl}_{2}$, (Litt.: ${ }^{19} 134{ }^{\circ} \mathrm{C}$, benzene/Et $\left.2 \mathrm{O}\right)$. TLC $\left(\mathrm{AcOEt} / \mathrm{CH}_{2} \mathrm{Cl}_{2}\right.$, 8:92) $R f=0.23 .{ }^{1} \mathrm{H}$ NMR $\left(400 \mathrm{MHz}, \mathrm{CDCl}_{3}\right): \delta 3.88$ and $3.93(\mathrm{AB}, J=13.0,2 \mathrm{H}), 7.27-7.41(\mathrm{~m}$, 10H). ${ }^{13} \mathrm{C}$ NMR $\left(62.9 \mathrm{MHz}, \mathrm{CDCl}_{3}\right): \delta: 57.4,128.4,129.0,130.23,130.25 . \mathrm{IR}\left(\mathrm{KBr}, \mathrm{cm}^{-1}\right): v$ 3060, 3030, 2956, 2926, 1030. MS (EI) m/z, $230\left(\mathrm{M}^{+}, 3\right), 91$ (100), 65 (21).

[(Cyclohexylsulfinyl)methyl $]$ benzene $2 \mathrm{~g} .{ }^{20}$ Obtained from $\beta$-sulfinylester $\mathbf{1 g}\left(\mathrm{R}^{1}=\right.$ cyclo$\mathrm{C}_{6} \mathrm{H}_{11}, \mathrm{R}^{2}=\mathrm{Et}, 242 \mathrm{mg}, 1.02 \mathrm{mmol}$ ) and benzyl bromide as the electrophile. Yield $76 \%$ (176 mg, 0.79 mmol). Orange solid. mp: $82-84{ }^{\circ} \mathrm{C}, \mathrm{CH}_{2} \mathrm{Cl}_{2} / \mathrm{AcOEt}$, (Litt.. ${ }^{20} 84-85^{\circ} \mathrm{C}$, benzene/petroleum ether). TLC $\left(\mathrm{CH}_{2} \mathrm{Cl}_{2} / \mathrm{AcOEt}, 90: 10\right) R f=0.27 .{ }^{1} \mathrm{H} \mathrm{NMR}\left(400 \mathrm{MHz}, \mathrm{CDCl}_{3}\right): \delta 1.20-1.35(\mathrm{~m}, 3 \mathrm{H})$, $1.46-1.59(\mathrm{~m}, 2 \mathrm{H}), 1.67-1.70(\mathrm{~m}, 1 \mathrm{H}), 1.79-1.95(\mathrm{~m}, 4 \mathrm{H}), 2.07-2.11(\mathrm{~m}, 1 \mathrm{H}), 2.45(\mathrm{tt}, J=3.5$ and $11.7,1 \mathrm{H}), 3.90$ and $3.98(\mathrm{AB}, J=13.0,2 \mathrm{H}), 7.28-7.39(\mathrm{~m}, 5 \mathrm{H}) .{ }^{13} \mathrm{C}$ NMR $(62.9 \mathrm{MHz}$, $\left.\mathrm{CDCl}_{3}\right): \delta 24.1,25.3,25.6,25.7,27.1,54.9,57.1,128.3,129.1,130.1,130.8 . \mathrm{IR}\left(\mathrm{KBr}, \mathrm{cm}^{-1}\right): v$ 3063, 3029, 2930, 2915, 2852, 1732, 1495, 1447, 1022. MS (EI) m/z $223\left(\mathrm{MH}^{+}, 36\right), 140$ (24), 91 (100).

[(t-Butylsulfinyl)methyl]benzene $2 \mathrm{~h} .{ }^{19}$ Obtained from $\beta$-sulfinylester $\mathbf{1 h}\left(\mathrm{R}^{1}=t\right.$-Bu, $\mathrm{R}^{2}=$ Et, $206 \mathrm{mg}, 1.00 \mathrm{mmol}$ ) and benzyl bromide as the electrophile. Yield 79\% (156 mg, $0.79 \mathrm{mmol})$. White solid. mp: $74-75^{\circ} \mathrm{C}\left(\mathrm{Litt.} .:^{19} 71{ }^{\circ} \mathrm{C}\right)$. TLC $\left(\mathrm{CH}_{2} \mathrm{Cl}_{2} / \mathrm{AcOEt}, 85: 15\right) R f=0.25 .{ }^{1} \mathrm{H}$ NMR $(400$ $\left.\mathrm{MHz}, \mathrm{CDCl}_{3}\right): \delta 1.33(\mathrm{~s}, 9 \mathrm{H}) 3.63$ and $3.83(\mathrm{AB}, J=12.9,2 \mathrm{H}), 7.33-7.37(\mathrm{~m}, 5 \mathrm{H}) .{ }^{13} \mathrm{C}$ NMR $\left(62.9 \mathrm{MHz}, \mathrm{CDCl}_{3}\right): \delta 23.4,53.4,54.1,128.4,129.2,130.4,132.5 . \mathrm{IR}\left(\mathrm{KBr}, \mathrm{cm}^{-1}\right)$ : v 3032, 2964 , 2926, 2866, 1034. MS (CI, isobutane) $m / z 235\left[\left(\mathrm{M}^{2} \mathrm{C}_{3} \mathrm{H}_{3}{ }^{+}\right), 3\right], 197\left(\mathrm{MH}^{+}, 100\right), 181(20), 154$ (46).

(20) O'Donnel, J. S.; Schwan, A. L. Tetrahedron Lett. 2003, 44, 6293-6296. 
Typical Procedure for Enantioenrichied Sulfoxides 2 with $n$-BuLi/(-)-Sparteine as Base: Alkylation of the Intermediate Sulfenate at $\mathbf{- 4 5}{ }^{\circ} \mathrm{C} . n-\mathrm{BuLi}(770 \mu \mathrm{L}$ of a $1.43 \mathrm{M}$ solution in hexanes, $1.1 \mathrm{mmol}, 1.1 \mathrm{eq}$ ) was added at room temperature to a solution of (-)-sparteine (234 $\mathrm{mg}, 1 \mathrm{mmol}, 1 \mathrm{eq})$ in toluene $(2 \mathrm{~mL})$. After being stirred for $15 \mathrm{~min}$, the resulting complex was added dropwise to a solution of $\beta$-sulfinylester $1 \mathbf{a}(240 \mathrm{mg}, 1 \mathrm{mmol}, 1 \mathrm{eq})$ in toluene $(5 \mathrm{~mL})$ previously cooled at $-45^{\circ} \mathrm{C}$. The reaction mixture was stirred at $-45^{\circ} \mathrm{C}$ for 30 min, treated with methyl iodide ( $65 \mu \mathrm{L}, 1 \mathrm{mmol}, 1 \mathrm{eq}$ ), stirred at this temperature for $4 \mathrm{~h}$ and quenched with a saturated aqueous $\mathrm{NH}_{4} \mathrm{Cl}$ solution $(20 \mathrm{~mL})$. The resulting mixture was extracted with ethyl acetate $(3 \times 25 \mathrm{~mL})$. The combined organic layers were successively washed with a saturated aqueous $\mathrm{NH}_{4} \mathrm{Cl}$ solution $(20 \mathrm{~mL})$, saturated aqueous $\mathrm{NaCl}$ solution $(10 \mathrm{~mL})$, dried over $\mathrm{MgSO}_{4}$, filtered and concentrated under reduced pressure. The resulting solid was then purified on silica gel eluting with diethyl ether to afford enantioenriched sulfoxide $\mathbf{2} \mathbf{a}_{\mathbf{2}}$ as a white solid (62 $\mathrm{mg}, 40 \%, 29 \%$ ee).

Results obtained for alkylation temperatures ranging from $-78{ }^{\circ} \mathrm{C}$ to $-20^{\circ} \mathrm{C}$ are given in the table below.

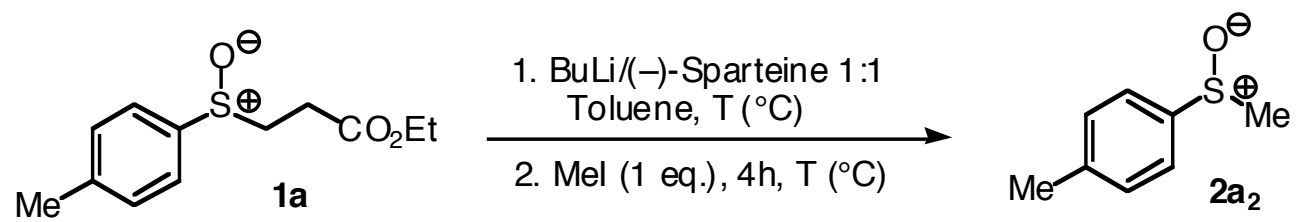

\begin{tabular}{|c|c|c|c|}
\hline Entry & $\mathrm{T}\left({ }^{\circ} \mathrm{C}\right)$ & Yield $(\%)$ & $\operatorname{Ee}(\%)^{\mathrm{a}}$ \\
\hline 1 & -78 & 14 & $18(S)$ \\
\hline 2 & -60 & 15 & $15(S)$ \\
\hline 3 & -50 & 37 & $23(S)$ \\
\hline 4 & -45 & 40 & $29(S)$ \\
\hline 5 & -40 & 54 & $23(S)$ \\
\hline 6 & -30 & 67 & $12(S)$ \\
\hline 7 & -20 & 69 & $9(S)$ \\
\hline
\end{tabular}

${ }^{a}$ The configuration of the major enantiomer is shown in parentheses. 


\section{HPLC Analysis}

Each enantiomer is characterized by a retention time $\mathbf{t}_{\mathbf{n}}(\mathrm{n}=1$ or 2$)$ and a capacity factor $\mathbf{k}_{\mathbf{n}}$ $(\mathrm{n}=1$ or 2$)$ calculated according to formula $(\mathbf{1}) \cdot \mathbf{t}_{\mathbf{0}}$ corresponds to the retention time of tertbutylbenzene, an apolar compound which has no interaction with the column. Selectivity factor $\alpha$ is calculated with formula (2). Resolution Rs, defining separation between two products, is calculated according to formula (3) in which $\omega$ is the breadth of a pic at the bottom.

$$
k_{n}=\frac{t_{n}-t_{0}}{t_{0}}
$$

(1) $\alpha=\frac{k_{1}^{\prime}}{k_{2}^{\prime}}$

(2)

$$
R s=\frac{2\left(t_{2}-t_{1}\right)}{\omega_{1}+\omega_{2}}
$$

(3)

Racemic 1-Benzylsulfinyl-4-methyl-benzene $2 \mathrm{a}_{1}$. AD-H column, $10{ }^{\circ} \mathrm{C}, 1 \mathrm{~mL} \cdot \mathrm{min}^{-1}, n$ -

\begin{tabular}{|c|c|c|c|c|c|}
\hline & $\begin{array}{l}\text { Retention time } \\
(\text { min) }\end{array}$ & $\lambda_{\max }(\mathrm{nm})$ & $\mathbf{k}^{\prime}$ & $\alpha$ & Rs \\
\hline$S_{S}$ & 17.32 & \multirow[t]{2}{*}{200.6} & 4.27 & \multirow[t]{2}{*}{1.15} & \multirow[t]{2}{*}{0.59} \\
\hline $\boldsymbol{R}_{S}$ & 19.41 & & 4.91 & & \\
\hline
\end{tabular}
heptane/i-PrOH 90:10.

Racemic 1-Methanesulfinyl-4-methyl-benzene $2 \mathbf{a}_{2}$. OB-H column, $20{ }^{\circ} \mathrm{C}, 1 \mathrm{~mL} \cdot \mathrm{min}^{-1}, n$ -

\begin{tabular}{|c|c|c|c|c|c|}
\hline & $\begin{array}{c}\text { Retention time } \\
\text { (min) }\end{array}$ & $\lambda_{\max }(\mathbf{n m})$ & $\mathbf{k}^{\prime}$ & $\alpha$ & Rs \\
\hline$S_{S}$ & 6.55 & \multirow{2}{*}{200.6} & 0.9 & \multirow{2}{*}{2.7} & \multirow{2}{*}{4.68} \\
\hline $\boldsymbol{R}_{S}$ & 11.83 & & 2.43 & & \\
\hline
\end{tabular}
heptane/ $i-\mathrm{PrOH}$ 70:30. 


\section{HPLC analysis of \\ racemic 1-benzylsulfinyl-4-methylbenzene $2 a_{1}$}

AD-H column

$10{ }^{\circ} \mathrm{C}, 1 \mathrm{~mL} \cdot \mathrm{min}^{-1}, n$-heptane $/ i$-PrOH 90:10

\section{Chromatogram}

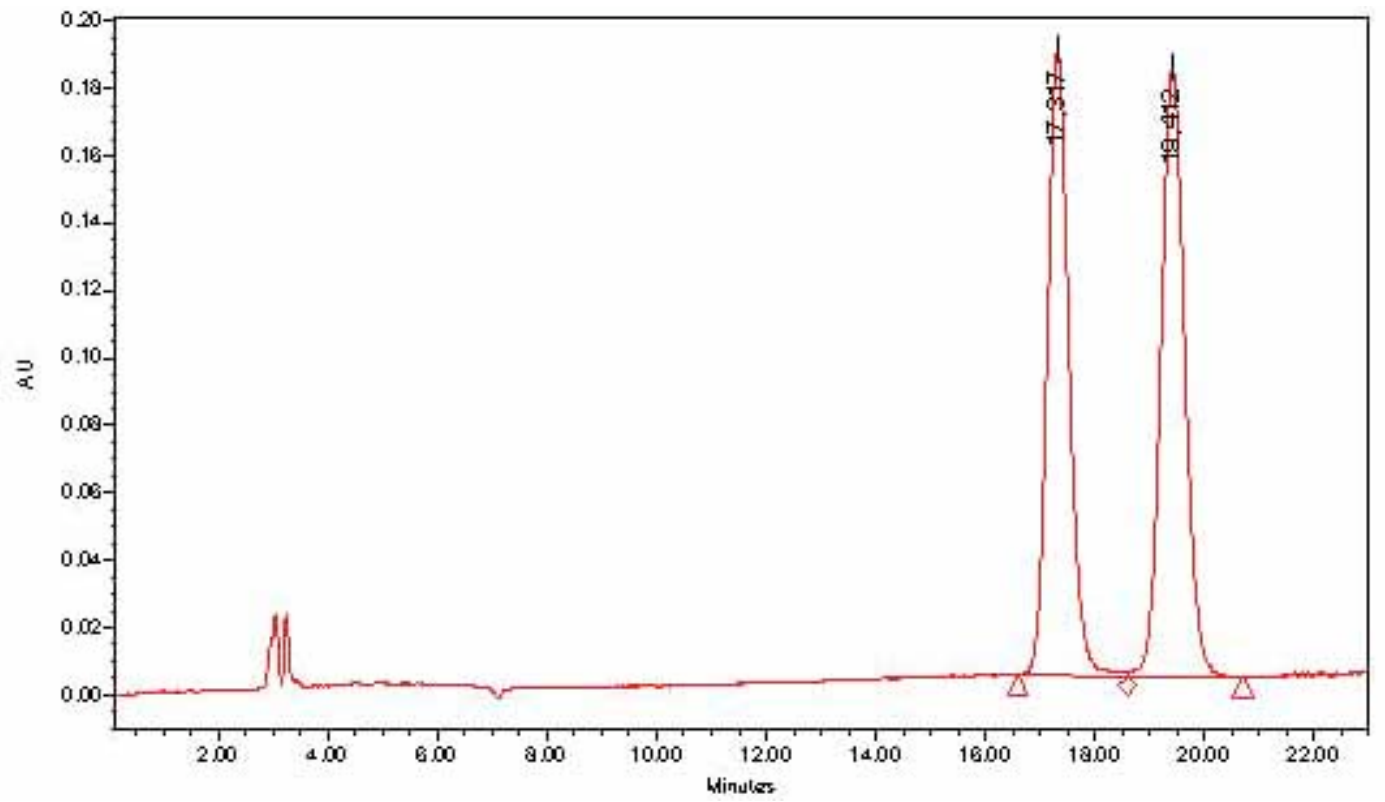

\section{UV Absorptions}

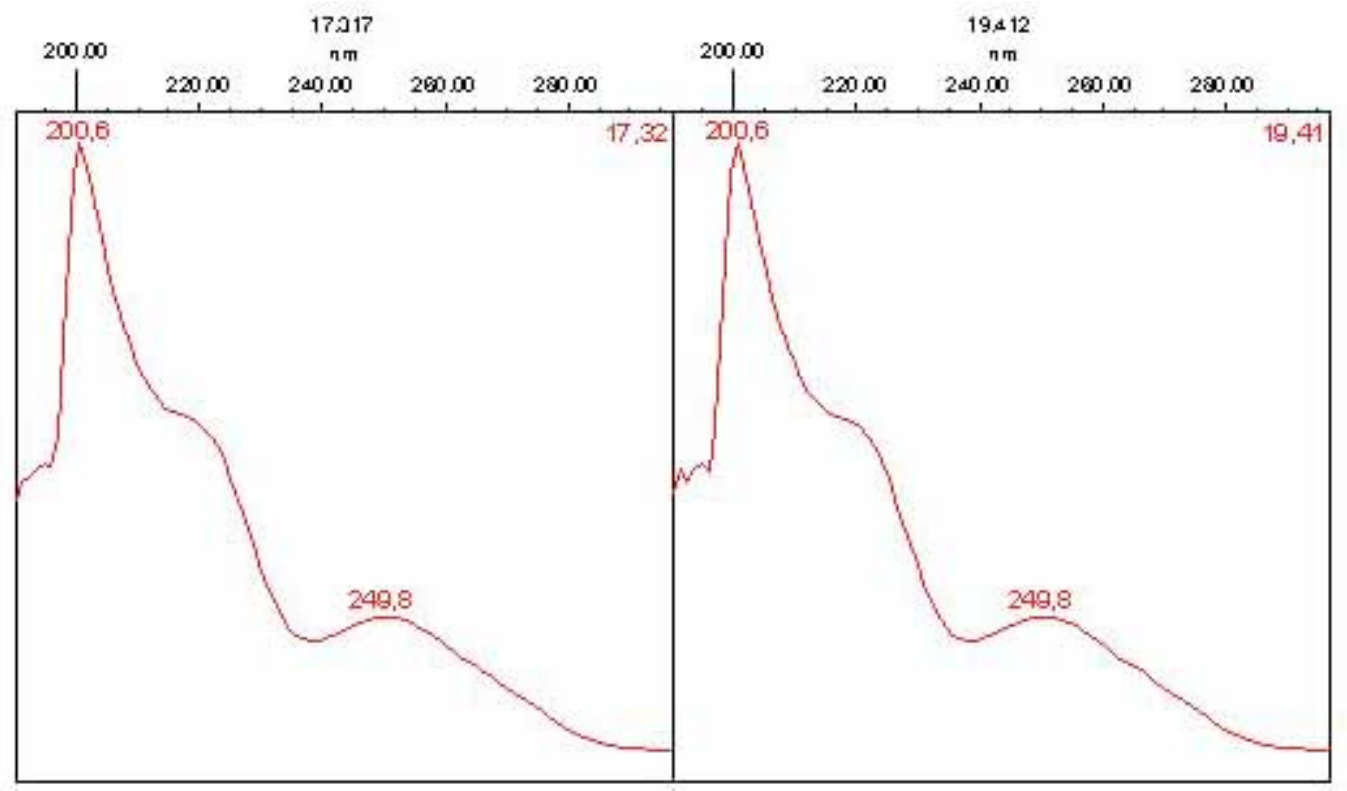




\title{
HPLC analysis of enantioenrichied sample of 1-benzylsulfinyl-4-methylbenzene $2 a_{1}$ : asymmetric thioether oxidation
}

\author{
AD column \\ $10{ }^{\circ} \mathrm{C}, 1 \mathrm{~mL} \cdot \mathrm{min}^{-1}$, hexane/i-PrOH 90:10 \\ $e e=75 \%(S)$
}

\section{Chromatogram}

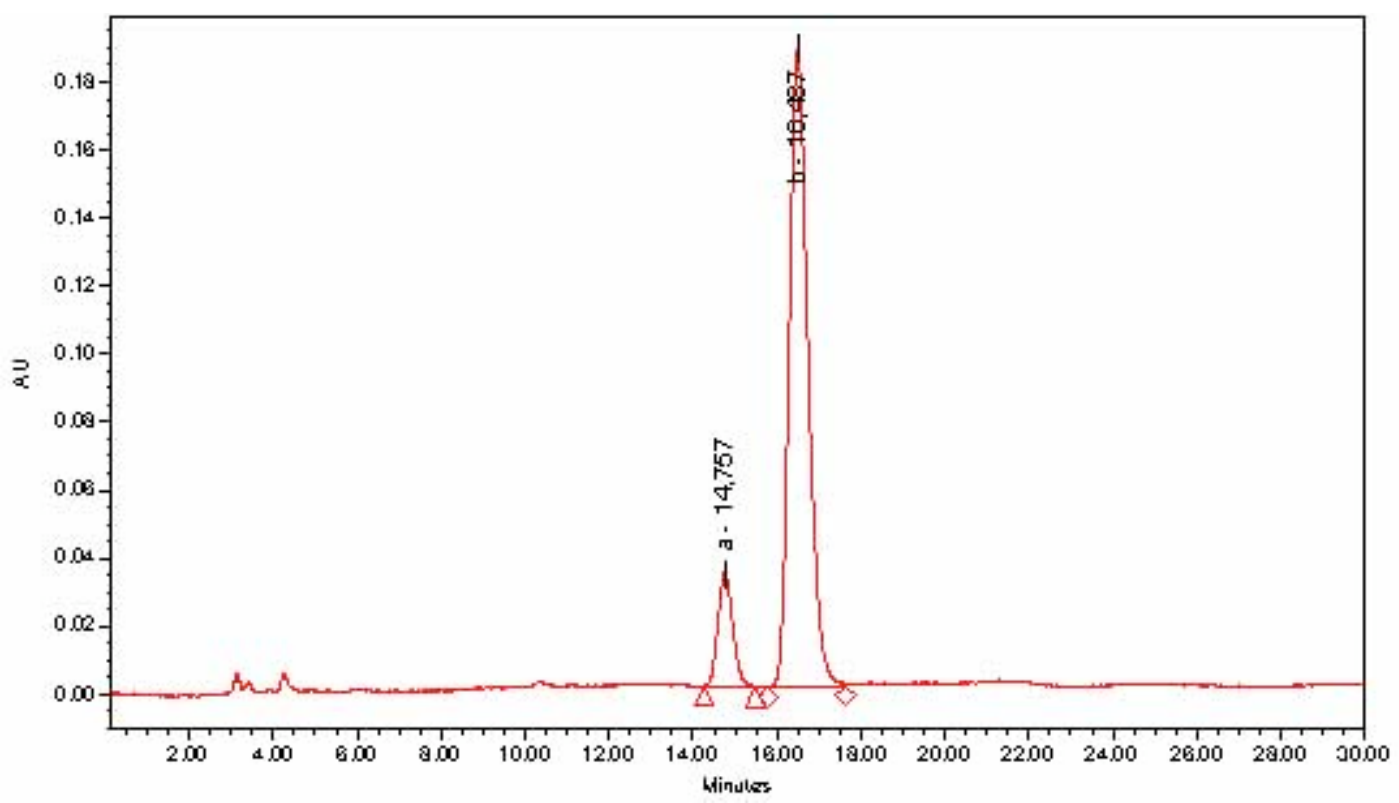

\section{UV Absorptions}

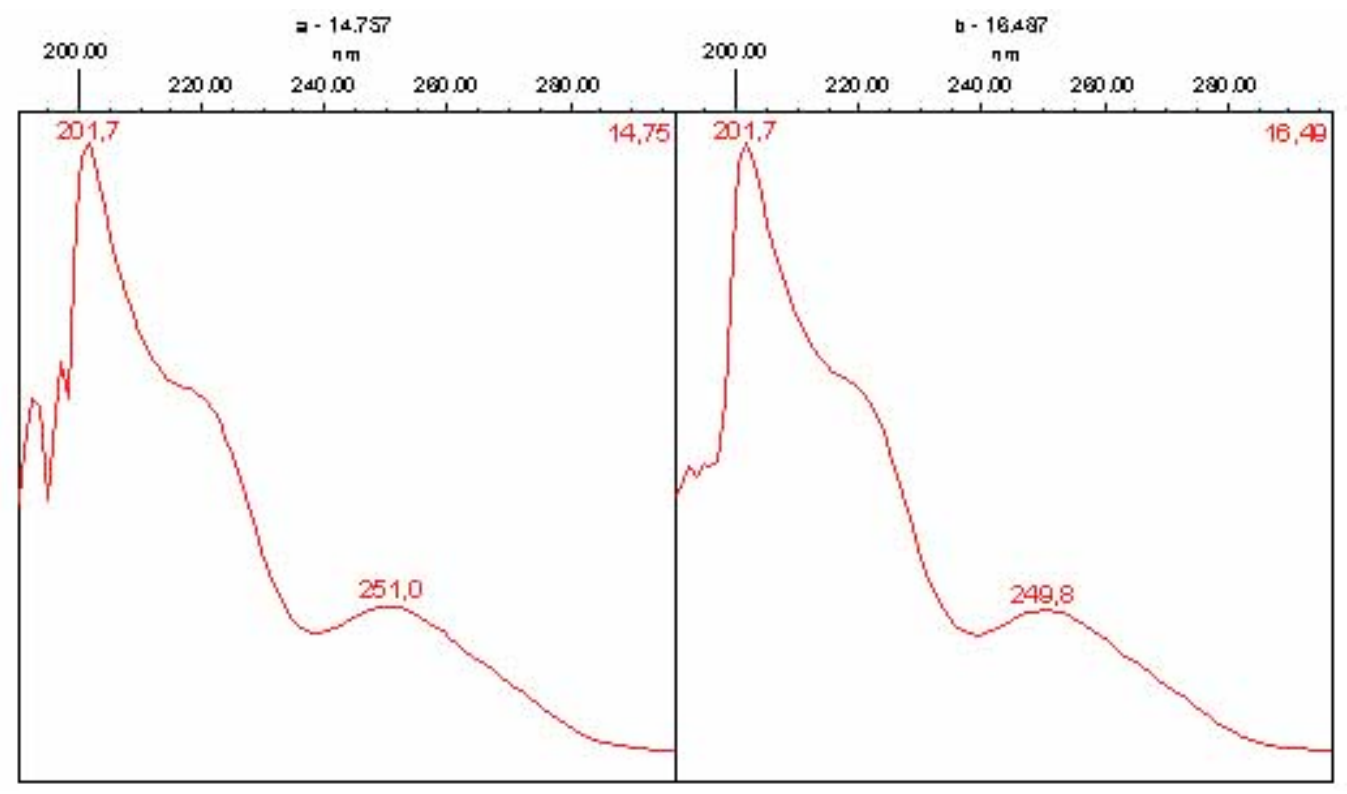




\section{HPLC analysis of}

enantioenrichied 1-benzylsulfinyl-4-methyl-benzene $2 \mathrm{a}_{1}$ : enantioselective alkylation of sulfenate in toluene at $-40{ }^{\circ} \mathrm{C}$

$$
\begin{gathered}
\text { AD-H column } \\
10^{\circ} \mathrm{C}, 1 \mathrm{~mL} \cdot \mathrm{min}^{-1}, n \text {-heptane } / i \text {-PrOH 90:10 } \\
\boldsymbol{e e}=\mathbf{1 7 \%}(\boldsymbol{S})
\end{gathered}
$$

\section{Chromatogram}

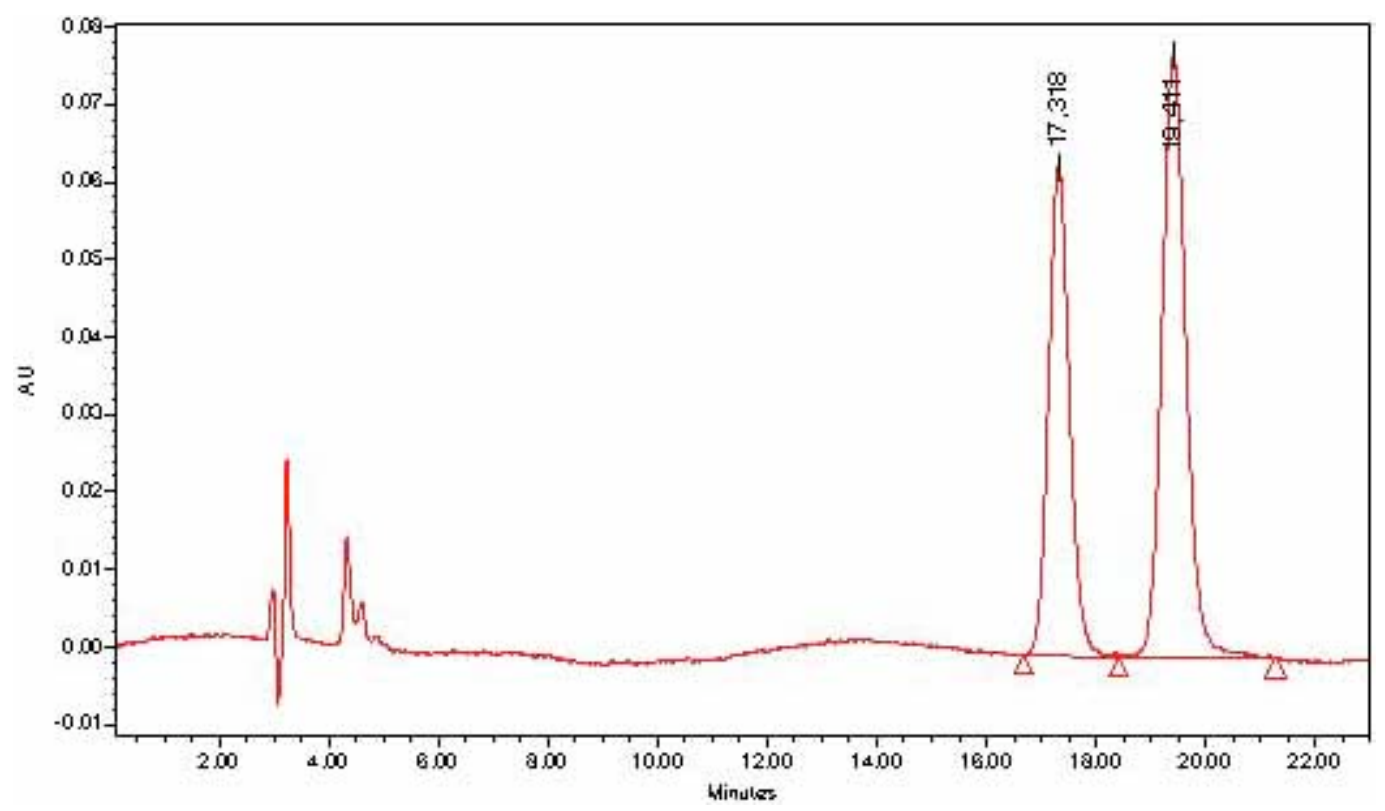

\section{UV Absorptions}

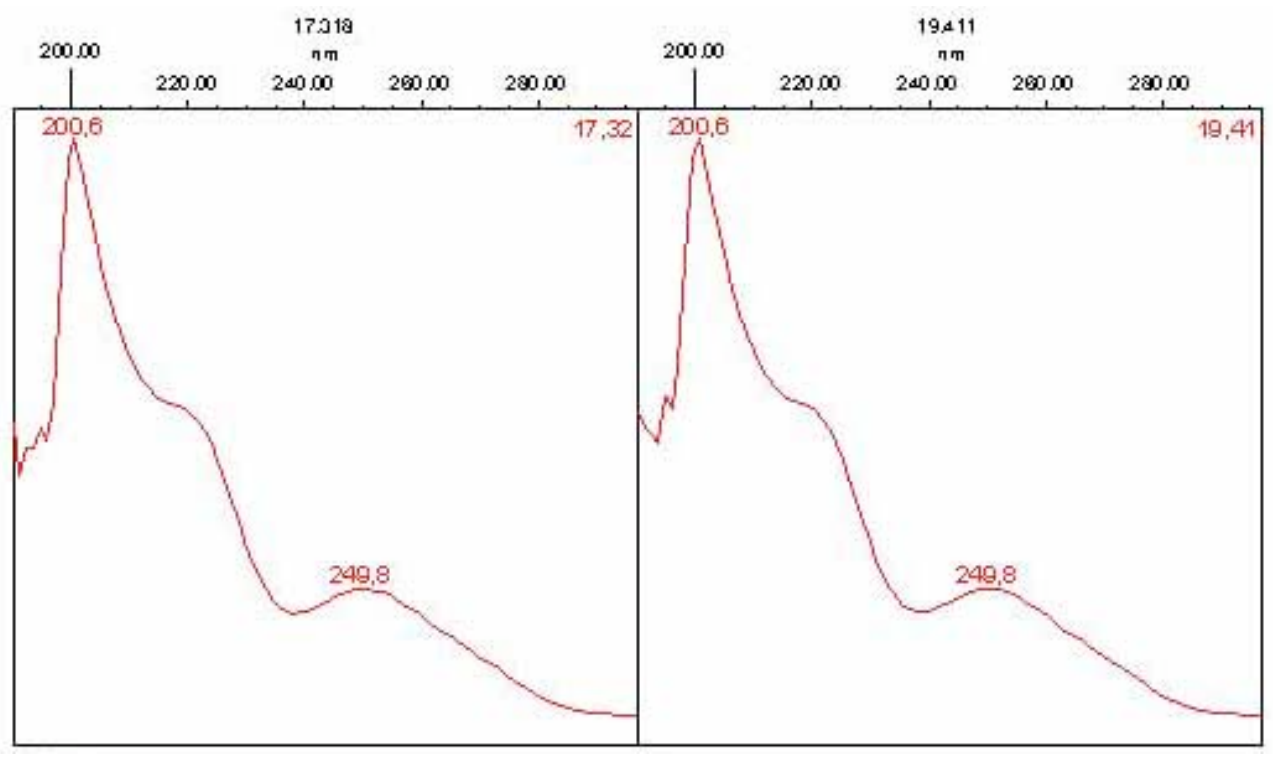




\section{HPLC analysis of racemic 1-methylsulfinyl-4-methylbenzene $2 \mathbf{a}_{2}$}

OB-H column

$20{ }^{\circ} \mathrm{C}, 1 \mathrm{~mL} \cdot \mathrm{min}^{-1}, n$-heptane $/ i$-PrOH 70:30

\section{Chromatogram}

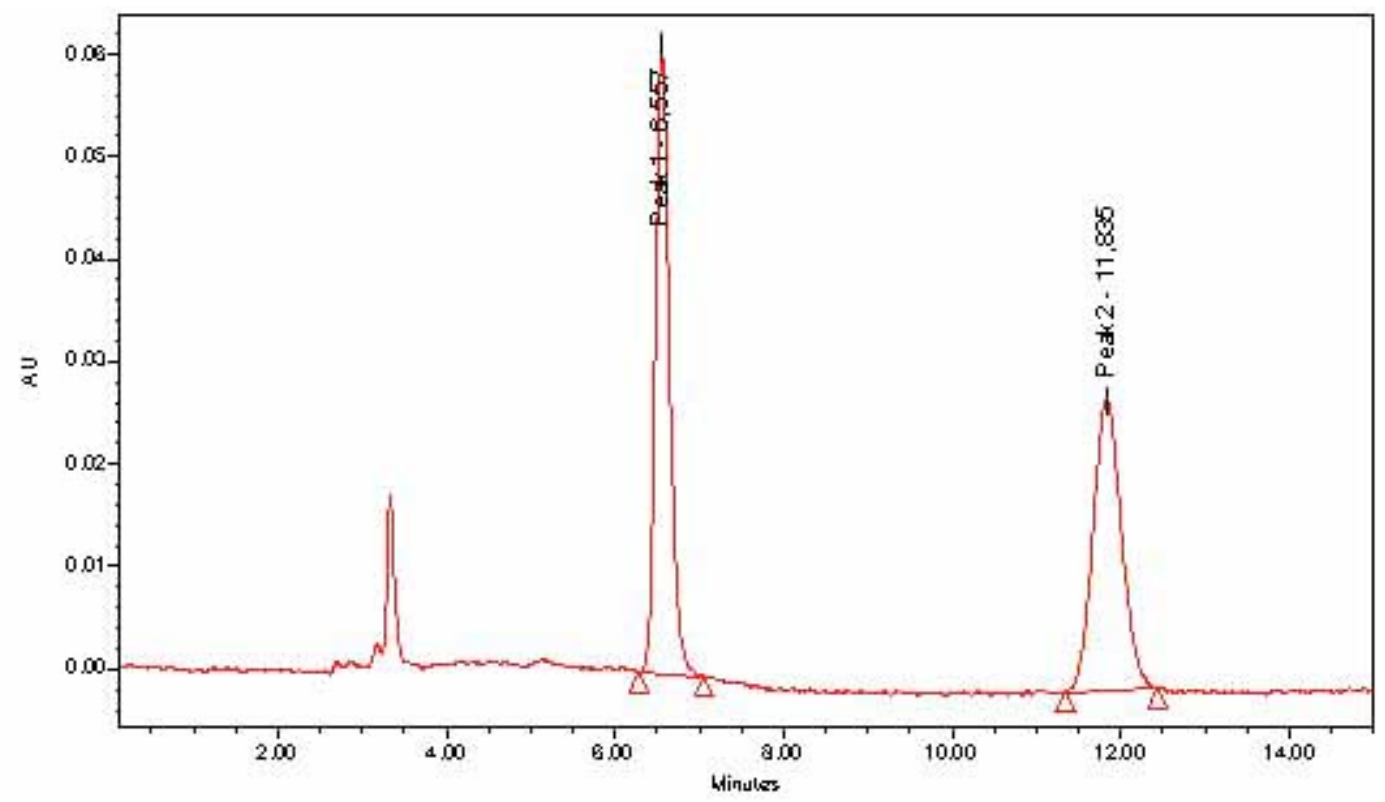

\section{UV Absorptions}

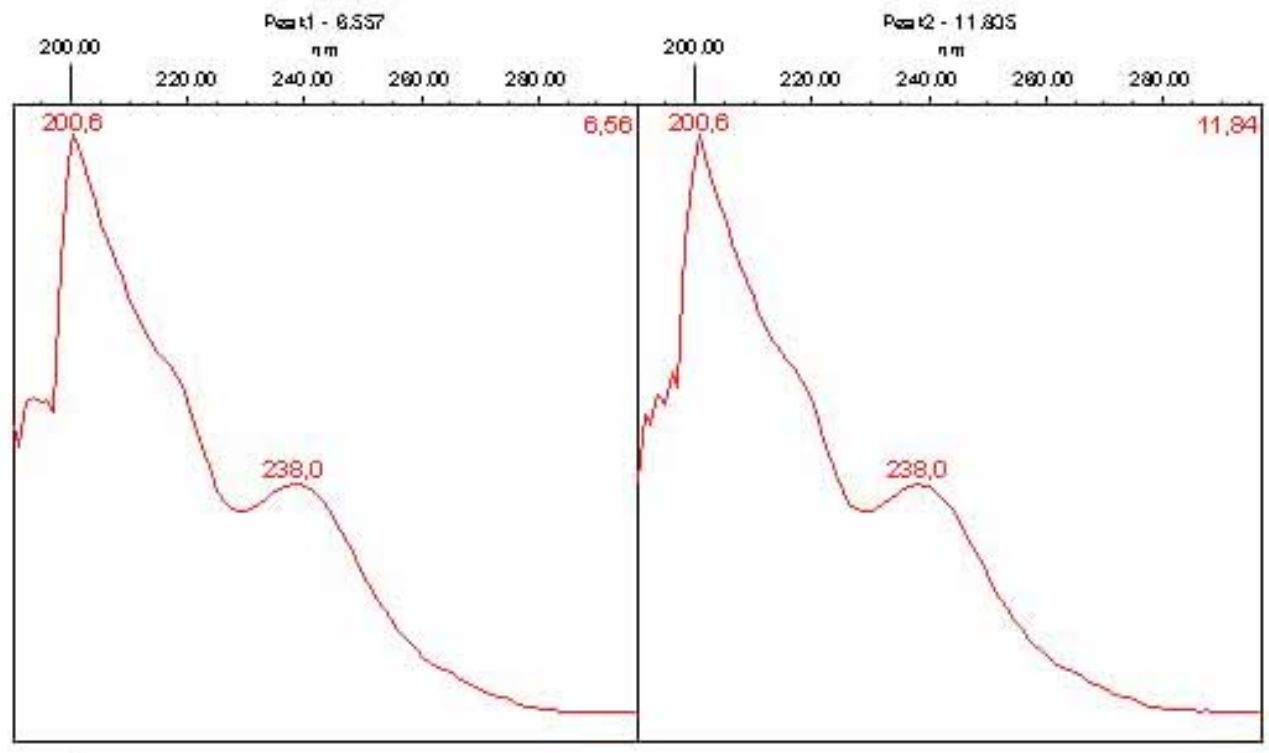


HPLC analysis of an enantioenrichied sample

of 1-methanesulfinyl-4-methylbenzene $2 \mathbf{a}_{2}$ : asymmetric thioether oxidation

$$
\begin{gathered}
\text { OB column } \\
20{ }^{\circ} \mathrm{C}, 1 \mathrm{~mL} \cdot \mathrm{min}^{-1}, \text { hexane } / i-\operatorname{PrOH} 80: 20 \\
\boldsymbol{e} \boldsymbol{e}=\mathbf{6 6} \%(\boldsymbol{S}), \text { litt }^{3 \mathrm{~b}}: \mathbf{6 7 \%}(\boldsymbol{S})
\end{gathered}
$$

\section{Chromatogram}

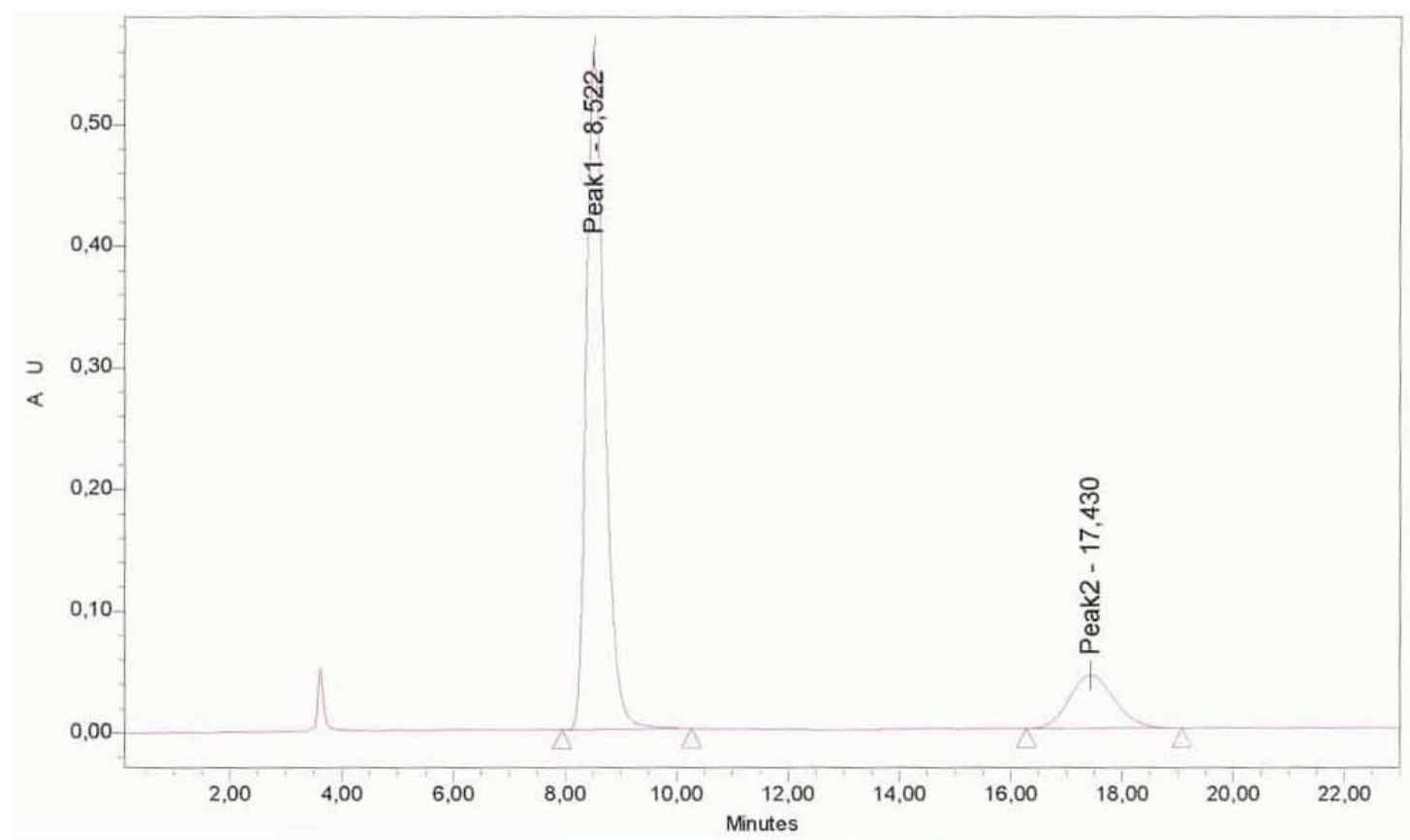

UV Absorptions

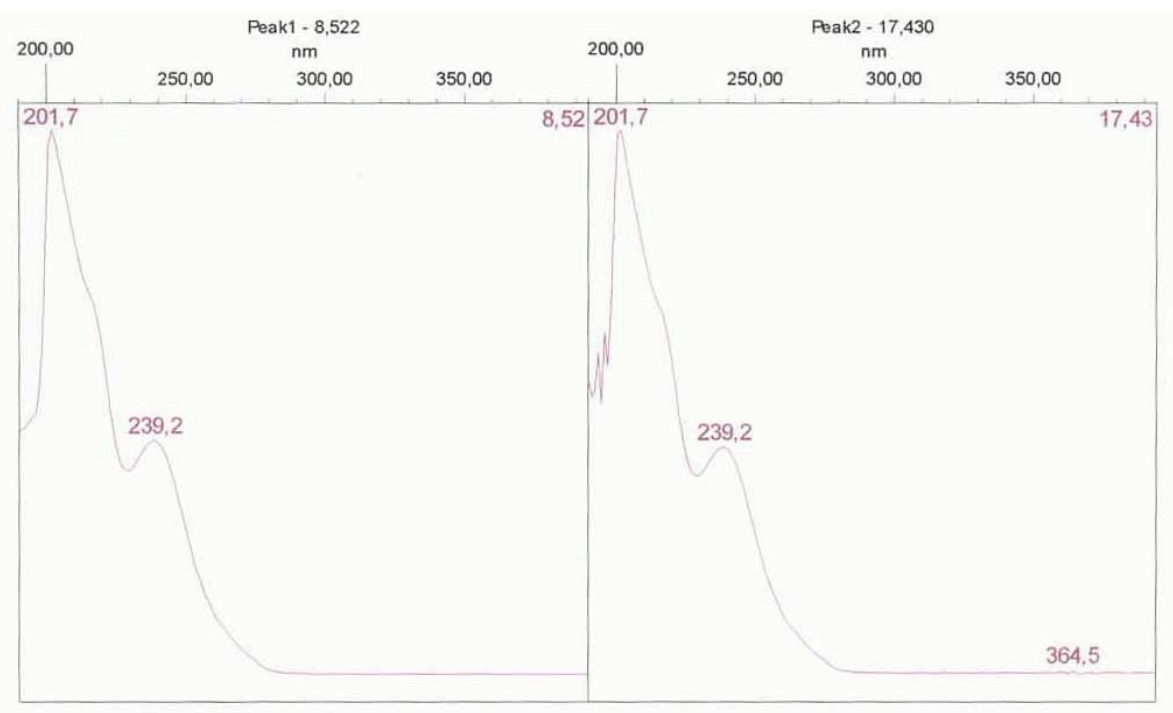


HPLC analysis of

enantioenrichied 1-methanesulfinyl-4-methylbenzene $2 \mathrm{a}_{2}$ : enantioselective alkylation of sulfenate in toluene at $-45^{\circ} \mathrm{C}$

OB-H column

$20{ }^{\circ} \mathrm{C}, 1 \mathrm{~mL} \cdot \mathrm{min}^{-1}, n$-heptane $/ i$-PrOH 70:30

$e e=29 \%(S)$

\section{Chromatogram}

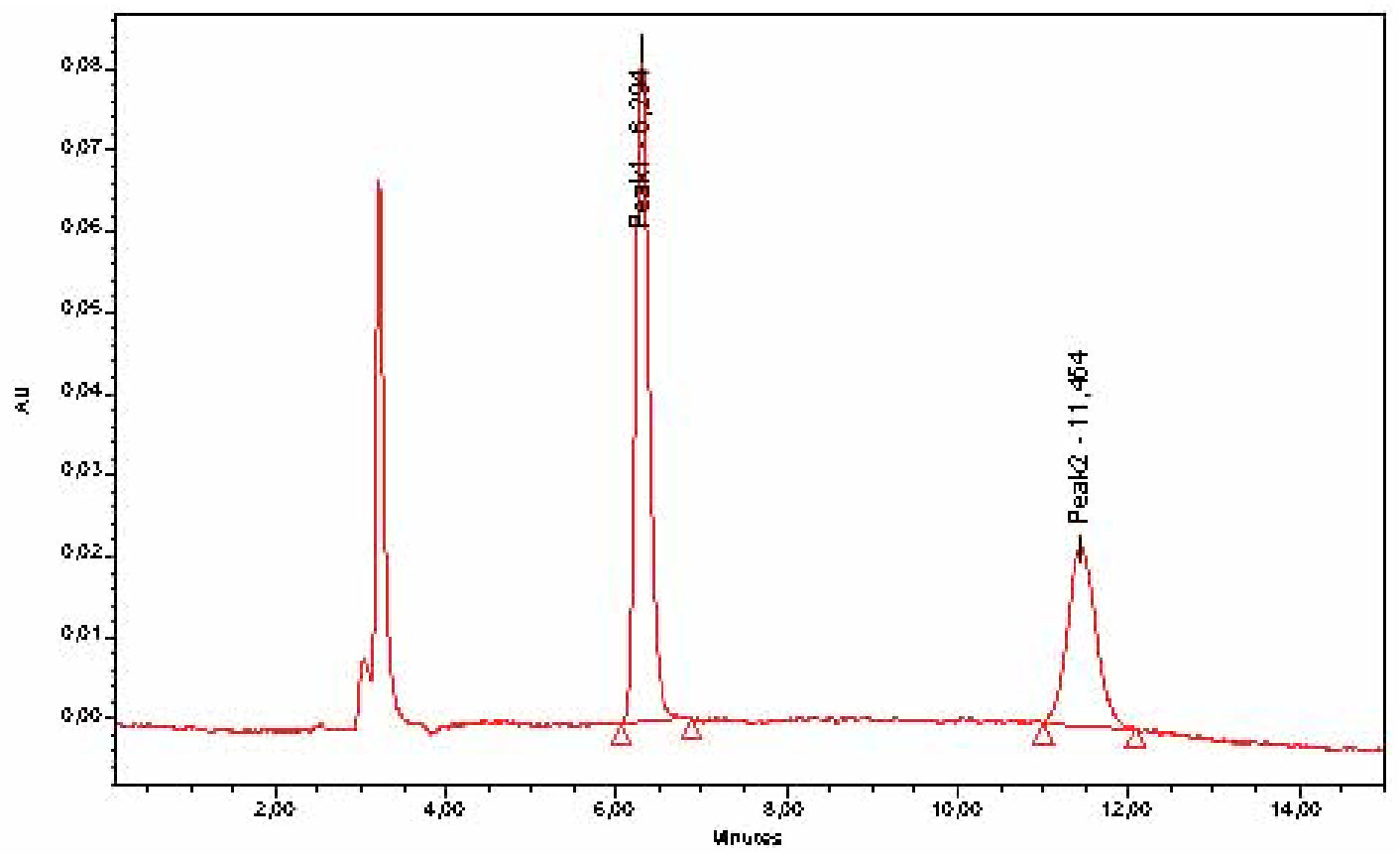

UV Absorptions

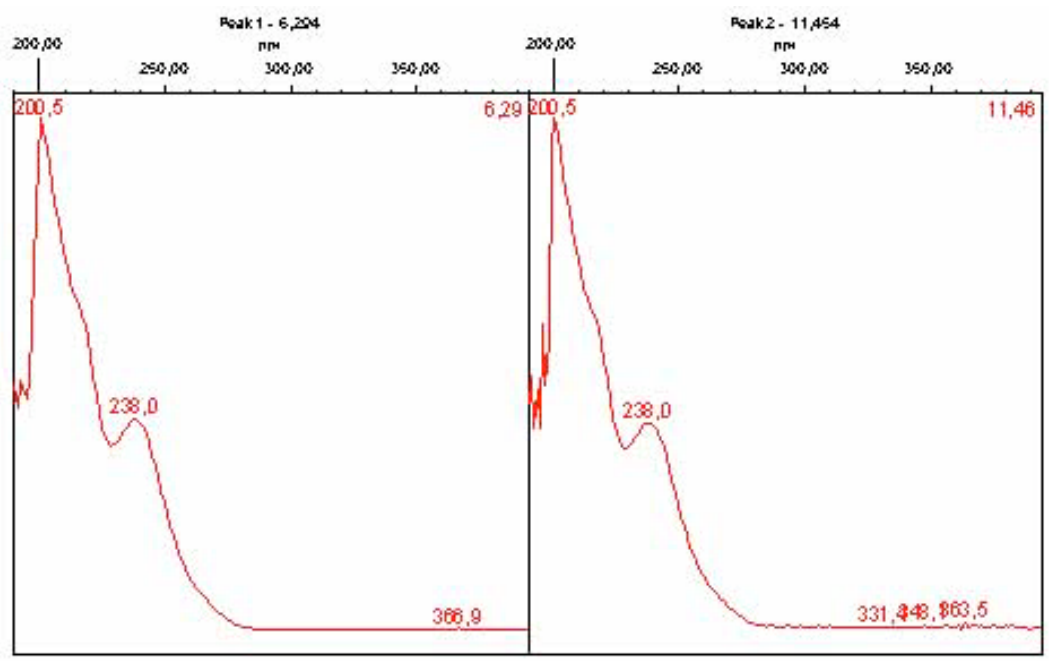

